\title{
MARX-GESAMTAUSGABE - DRINGENDES PARTEIINTERESSE ODER DEKORATIVER ZWECK?
}

\author{
EIN WIENER EDITIONSPLAN ZUM 30. TODESTAG, BRIEFE \\ UND BRIEFAUSZÜGE
}

Ende 1910 machte man sich in Wien Gedanken darüber, wie sich die Sozialdemokratie auf den dreißigsten Todestag von Karl Marx vorbereiten solle. Gustav Eckstein berichtete Karl Kautsky am 27. Dezember:

Für Freitag Abend bin ich zu einer „,Marxisten-Konferenz” geladen, bei der die Situation besprochen werden soll, die sich aus der Freigabe von Marx' Schriften ergeben wird. Braun teilte mir mit, daß geplant sei, entweder eine internationale Aktion oder wenigstens eine der deutschen Marxisten zur Neuherausgabe von Marx' Schriften einzuleiten; und da Hilferding und ich jetzt gerade hier sind, soll Freitag eine Konferenz darüber stattfinden, an der Goldendach, Renner, Max Adler, Braun, Bauer, Hilferding u. ich teilnehmen sollen. Natürlich erwähnte ich nichts von Dietz' Plan und werde davon auch ohne Ihre Einwilligung nichts erwähnen. Wie mir Braun sagte, hat er den Plan, Sie zu fragen, ob es Ihnen recht wäre, daß ein Kommittee von Marxisten an Sie einen offenen Brief schreiben und die Anregung zur Neuherausgabe geben [wird]. Näheres weiß ich noch nicht, das soll eben erst Freitag besprochen werden. ${ }^{1}$

Die fünf österreichischen Konferenzteilnehmer sind als Exponenten des Austromarxismus bekannt. ${ }^{2}$ Sie bilden nach Alter, sozialer Herkunft und akademischer Vorbildung eine recht homogene Gruppe ${ }^{3}$ seit der Jahr-

1 G. Eckstein an K. Kautsky, 27.12.1910, Nachlaß Kautsky D X 56, Internationaal Instituut voor Sociale Geschiedenis. Gustav Eckstein (1875-1916), sein Hintergrund und Werdegang sind denen der von ihm genannten Austromarxisten vergleichbar (s. Anm. 3), er war seit 1902 regelmäßiger Mitarbeiter der Neuen Zeit, ab 1910 Redaktionsmitglied und für die Krankheitsvertretung Kautskys vorgesehen; vgl. Bebel an L. Kautsky, 1.11.1910, in August Bebels Briefwechsel mit Karl Kautsky, hrsg. von K. Kautsky Jr (Quellen und Untersuchungen zur Geschichte der deutschen und österreichischen Arbeiterbewegung, Neue Folge, Bd 2), Assen 1971, S. 240. Zu „Dietz' Plan” einer Volksausgabe von K. Marx, Das Kapital, Bd I, vgl. unten, S. 117.

2 Vgl. z.B. E. Glaser, Im Umfeld des Austromarxismus. Ein Beitrag zur Geistesgeschichte des österreichischen Sozialismus, Wien usw. 1981, S. 17f. und passim.

3 Max Adler (1873-1937), Otto Bauer (1881-1938), Adolf Braun (1862-1929), Rudolf Hilferding (1877-1941), Karl Renner (1870-1950); alle außer Braun sind seit 1870 geboren, alle außer dem Kleinbauernsohn Renner entstammen dem mehr oder weniger 
hundertwende gelten sie - von Kautsky freudig begrüßt - als ,,marxistischer Nachwuchs". 4

Mit den Marx-Studien hatte sich die Gruppe 1904 ein wissenschaftliches Forum geschaffen, Adler und Hilferding fungierten als Herausgeber. Titelwahl und Programm sollten keinen Zweifel über die Frontstellung gegen den Revisionismus lassen und wurden von Bernstein auch durchaus als Kriegserklärung aufgefaßt. ${ }^{5}$ Zugleich verstand man sich ausdrücklich als Marxist der zweiten Generation und ging vorsichtig auf Distanz zur Arbeitsweise der älteren Orthodoxie, die sich zu den einmal von Marx und Engels erreichten Resultaten primär popularisierend und apologetisch verhalten habe. Das eigene Arbeitsinteresse richtete man auf sozialwissenschaftliche und philosophische Forschung mit an Marx orientierter Fragestellung in Bereichen, die dieser noch nicht bearbeitet hatte. ${ }^{6}$ In Kautskys Neuer Zeit wurde das nicht viel anders gesehen, zusätzlich sind die historischen Bedingungen solcher Akzentverschiebung benannt. Die ältere Orthodoxie habe noch unter der Leitung von Engels für Verbreitung und Sicherung des Marxismus in der erstarkenden Arbeiterbewegung derart gesorgt, daß eine jüngere Generation der Intelligenz sich ohne ständige Rücksicht auf unmittelbare theoretische Bedürfnisse der Arbeiterbewegung mit den durch die seitherige Wissenschaftsentwicklung aufgeworfenen methodologischen und erkenntnistheoretischen Fragen im Marxschen Sinne auseinandersetzen könne. ${ }^{7}$ Der Kampf, den Bauer, Braun und Renner seit 1907 für die deutschösterreichische Sozialdemokratie herausgaben, widmete sich eben diesen unmittelbaren theoretischen Bedürfnissen mit kaum geringeren Ambitionen. Folgt man dem Programmartikel, so hatte im Rahmen einer internationalen Arbeitsteilung in der sozialistischen Bewegung die österreichische Sozialdemokratie in aller

begüterten jüdischen Bürgertum der Doppelmonarchie, alle haben Hochschulausbildung. Für die biographischen Rahmendaten vgl. Dictionnaire biographique du mouvement ouvrier international, hrsg. von J. Maitron und G. Haupt, Bd 1: Autriche, Paris 1971.

4 K. Kautsky an V. Adler, 25.10.1901 und 8.12.1904, in: Victor Adler, Briefwechsel mit August Bebel und Karl Kautsky [...], hrsg. von F. Adler, Wien 1954 (weiter AdlerBriefwechsel). S. 375 und 439. Braun, der schon in den 1880-er Jahren politisch tätig wurde, ist hier die Ausnahme.

5 „Vorwort”, in: Marx-Studien. Blätter zur Theorie und Politik des wissenschaftlichen Sozialismus, hrsg. von M. Adler und R. Hilferding, Bd 1, Wien 1904. S. vif., und Bernsteins Rezension des Bandes in Dokumente des Sozialismus, Bd 4 (1904). S. 153-58, bes. S. 154.

6 "Vorwort", a.a.O.

7 M. Zetterbaum, ,Die Marx-Studien”, in: Neue Zeit, Jg. 23 (1904-05). Bd 1, S. 196-204. 242-47, bes. S. 197-99; Zetterbaum stand Herausgebern und Autoren der Marx-Studien anscheinend nahe (vgl. Adler-Briefwechsel, a.a.O.), die Reaktion ist in den genannten Passagen weniger zurückhaltend, als Glaser, a.a.O., S. 28, sie liest. 
Bescheidenheit nicht mehr und nicht weniger zu erforschen und theoretisch zu bewältigen, als die Bedingungen der Einheit der Arbeiterbewegung unter national, sozio-kulturell, staatsrechtlich und ökonomisch extrem differierenden Bedingungen. Gelöst werden sollten die Existenzfragen der II. Internationale. ${ }^{8}$

Diese Kombination von hohen wissenschaftlichen und politischen Ansprüchen, die 1910 schon einige Jahre in enger Zusammenarbeit und nicht ohne Erfolg vertreten worden waren, ist zu berücksichtigen, wenn man die Unbefangenheit beobachtet, mit der sich die Herausgeber von MarxStudien und Kampf in's Feld der Marx-Edition begaben.

Den spezialisierten Sachverstand konnte Goldendach, d.h. Rjazanov, auf der Konferenz vertreten. ${ }^{9}$ Für nichtrussische Leser hatte er im März 1909 mit einer kritischen Studie zu Marxens Vorstellungen über die Rolle Rußlands in der neueren Geschichte Europas als Marx-Forscher „debütiert". ${ }^{10}$ An Forschungs- und Editionsvorhaben, die ihn in den Jahren 1909-10 beschäftigten, läßt sich eine Art Blitzkarriere ablesen. Geplant war eine Übersetzung der englischen Edition von Marxens Artikeln über den Krimkrieg, ${ }^{11}$ die Luise Kautsky besorgen sollte; es spricht vieles dafür, daß Rjazanov die wissenschaftliche Betreuung dieses Vorhabens im März 1909 schon übernommen hatte. Der Auftrag, den ungeschlagenen Bestseller der sozialdemokratischen Literatur, Bebels Frau, für die Jubiläumsausgabe zu bearbeiten, kann kaum viel später erfolgt sein. Beide Projekte beschäftigten Rjazanov im Sommerhalbjahr 1909 in London. ${ }^{12}$ Die Jubiläumsausgabe erschien wie vorgesehen. ${ }^{13}$ Dagegen fiel die Marx-Übersetzung vorläufig Rjazanovs Forschungseifer zum Opfer; die Durchsicht der

8 „Der Kampf”, in: Der Kampf. Sozialdemokratische Monatsschrift. Hrsg.: O. Bauer, A. Braun, K. Renner, Jg. 1 (1907-08), S. 1-5, bes. S. 3-5.

9 David Borisovič Gol'dendach / N. bzw. D. Rjazanov (1870-1938), eine Biographie des russischen Sozialdemokraten, sozialdemokratischen Marx-Forschers und späteren Begründers und Organisators der sowjetischen Marx-Forschung und -Edition steht aus. Zur Orientierung vgl. D. J. Struik, ,Introduction”, in: D. Riazanov, Karl Marx and Friedrich Engels. An introduction to their lives and works [. . . ], New York usw. [1973], S. 3-10.

$10 \mathrm{~N}$. Rjasanoff, Karl Marx über den Ursprung der Vorherrschaft Rußlands in Europa. Kritische Untersuchungen (Ergänzungshefte zur Neuen Zeit, Bd 5), Stuttgart 1909.

${ }^{11}$ K. Marx. The Eastern Question. A reprint of letters written 1853-1856 [. . .]. hrsg. von E. Marx Aveling und E. Aveling, London 1897. Zur geplanten deutschen Ubersetzung vgl. Rjasanoff, a.a.O., S. 8.

12 Die undatierten Briefe Rjazanovs an Luise und Karl Kautsky aus dem Sommerhalbjahr 1909 sind faktisch ausführliche Arbeitsberichte an die Mitarbeiterin und Übersetzerin, vgl. bes. (in dieser Folge) Rjazanov an L. bzw. K. Kautsky, s.d., Nachlaß Kautsky D XIX 282, 300, 280, 284, 285.

13 A. Bebel, Die Frau und der Sozialismus. Fünfzigste Auflage. Verbessert, vermehrt und neu bearbeitet. Jubiläumsausgabe, Stuttgart 1910; zu Rjazanovs Mitwirkung vgl. S. xxxif. 
amerikanischen und englischen Zeitungen ergab eine derartige Fülle von zusätzlichem Material, daß man die Eastern Question als Übersetzungsgrundlage fallen ließ und Rjazanov sich auf eine „Herausgabe des englischen Marx'schen Nachlasses, zugleich eine Vorgeschichte der Internationale" umorientierte. ${ }^{14}$ Eine Edition der Quellen der Internationalen Arbeiterassoziation, die die Wiener Menger-Stiftung ${ }^{15}$ veröffentlichen wollte, hatte Adolf Braun zunächst Karl Kautsky angetragen. Der lehnte ab und schlug im März 1909 Rjazanov als Bearbeiter vor, der den Auftrag im Juni erhielt. ${ }^{16}$ Dieses Urkundenbuch der Internationale gehört insofern in den Bereich der Marx-Edition, als es nach Rjazanovs Vorstellungen auch alle „Kundgebungen von Marx und Engels, die in die Jahre 1864-1876 fallen und die sich an ihre Tätigkeit in der Internationalen Arbeiterassoziation knüpfen", enthalten sollte. ${ }^{17}$

Geheimnistuerei, die Braun apostrophiert ( $\mathrm{Nr} 5$ ), umgab damals den Briefwechsel zwischen Marx und Engels, den Bernstein zur Edition vorbereitete. ${ }^{18}$ Nichts kennzeichnet die Vertrauensstellung, die Rjazanov in-

${ }^{14}$ Rjazanov an L. und K. Kautsky, [vor dem 25.12.1909,] Nachlaß Kautsky D XIX 301. Von der auf vier Bände konzipierten Ausgabe erschienen nur zwei: Gesammelte Schriften von K. Marx und F. Engels 1852-1862, hrsg. von N. Rjasanoff, die Übers. aus dem Engl. von L. Kautsky, Bd 1-2, Stuttgart 1917; zur Geschichte der Edition vgl. auch Rjazanovs Vorwort, Bd 1, bes. S. IX-XI.

${ }^{15}$ Anton Menger (184l-1906), von Engels und Kautsky als „Juristensozialist” a postrophiert, hinterließ sein Vermögen der Stiftung „Anton Menger-Bibliothek” mit der Aufgabe, ,,die Originalschriften älterer Autoren, die für die Volkssache eingetreten sind, in kritischen. streng wissenschaftlichen Neudrucken zu reproduzieren". Vgl. C. Grünberg, "Menger, Anton", in: Biographisches Jahrbuch und Deutscher Nekrolog, Bd 11 (1906), Berlin 1908, S. 3-22, bes. 13. Mit Carl Grünberg als Sekretär, Adolf Braun und Ludo M. Hartmann als Kuratoriumsmitgliedern, befand sich die Stiftung anscheinend ,fest in sozialdemokratischer Hand"; vgl. Dictionnaire biographique du mouvement ouvrier international, a.a.O., S. 117, und A. Braun an K. Kautsky. 28.4.1908, Nachlaß Kautsky D VI 290.

${ }^{16}$ Braun an Kautsky, 28.4.1908; ebenso, 29.3 und 22.6.1909. Nachlaß Kautsky D Vi 290, 296,300 .

${ }^{17}$ Vgl. Gesammelte Schriften von K. Marx und F. Engels, a.a.O., Bd I, S. IX. Ursprünglich war eine kommentierte Edition der Kongreßprotokolle beabsichtigt (Braun an Kautsky, 28.4. 1908), Rjazanovs erweiterter Plan lautete: „Das Urkundenbuch der Internationale / Annalen, Regesta, Bibliographie / Das heißt, ich schildere die Tätigkeit der Internationale nach den Dokumenten, Protokollen, Aufrufen, etc. etc., versehe das Ganze mit Anmerkungen und Kommentar und schiebe zwischen verschiedenen Dokumenten meine ,objektive' Interpolationen. Dabei werde ich alle Briefe, Generalratsverhandlungen etc. vollinhaltlich oder in Auszügen utilisieren. Der Ausgangspunkt bildet der Meeting 28. Sept. 1864 und die Darstellung wird bis in den Haager Kongreß fortgeführt mit einem kleinen Anhang bis 1876. dem Jahre des offiziellen Todes der Internationale." Rjazanov an L. und K. Kautsky, [vor dem 25.12.1909,] a.a.O.

${ }_{18}$ Der Briefwechsel zwischen Friedrich Engels und Karl Marx 1844 bis 1883, hrsg. von A. Bebel und E. Bernstein, 4 Bde, Stuttgart 1913. Für die Vertraulichkeit der Editionsarbeiten anschaulich Victor Adlers Bemühung um Einsichtnahme in den Brief- 
zwischen besaß, besser als die Tatsache, daß er diesen im September 1910 einsehen konnte. Wenig später geriet das Editionsvorhaben in seine erste Krise, und Mehring adressierte den Entwurf eines Rundschreibens mit seinen Lösungsvorschlägen an ,die Genossen Bebel, Bernstein, Dietz, Goldendach und Frau Lafargue". Rjazanovs Zugehörigkeit zum Kreise derer, die in Fragen der Marx-Edition mitredeten, ist dort notifiziert. $\mathrm{Zu}$ diesem Kreis hat sich Rjazanov, was Anspruch und Inventivität anging, verhalten wie seine Wiener Altersgenossen zur älteren Orthodoxie, der er aber als russischer Sozialdemokrat der ersten Generation in seinen politischen Reaktionsweisen wahrscheinlich näher stand als jene. ${ }^{19}$

Das Beratungsergebnis der Marxistenkonferenz war der hier veröffentlichte Wiener Editionsplan ( $\mathrm{Nr}$ 1). Er ist als Vorschlag an den Vorstand der SPD formuliert und wirkt in seinen inhaltlichen und in seinen organisatorischen Komponenten wie ein „Grand Dessein” zukünftiger sozialdemokratischer Marx-Edition.

Inhaltlich gesehen sollen wissenschaftliche Editionstätigkeit und solche für die Zwecke der Arbeiterbildung und Propaganda als gleichwichtige Aufgaben im Rahmen eines langfristigen Programms aufeinander abgestimmt werden. Die Art, wie die wissenschaftliche Gesamtausgabe der Werke von Marx in den Mittelpunkt des Plans gerückt wird, läßt aber keinen Zweifel über die wirkliche Gewichtsverteilung zu. Welchen

wechsel im März 19II (an Bebel, 19.3.1911, und dessen Antwort vom 22.3, in: AdlerBriefwechsel, S. 524-28). Die komplizierte Entstehungsgeschichte verdient eine eigene Darstellung, soweit sie bis Ende 1910 in unserem Zusammenhang relevant ist, wird sie weiter unten skizziert. Zur Konstellation in der Schlußphase, bis hin zum Versuch Kautskys, die Publikation noch im Mai 1913 zu verhindern oder aufzuschieben, vgl. Adler-Briefwechsel, S. 564-72. Das Resumé von Dietz (ebd., S. 569f.) betont die persönlich komplizierenden Komponenten, nach Erscheinen haben die Hauptkontrahenten ihre sachlich differierenden Positionen im Rahmen der Parteiloyalität formuliert: F. Mehring, ,Engels und Marx”, in: Archiv für die Geschichte des Sozialismus und der Arbeiterbewegung, Jg. 5 (1915), S. 1-38, und N. Rjasanoff, „Der Briefwechsel zwischen Marx und Engels", in: Neue Zeit, Jg. 32 (1913-14), Bd 2, S. 564-71. Rjazanovs Moskauer Akademievortrag von 1923 bietet eine, der veränderten Parteikonstellation entsprechend, ,gestraffte" Version, in der allerdings Bernsteins Verantwortlichkeit für die Streichungen in der Edition eher indirekt nahegelegt als behauptet wird, der Gegensatz mit Mehring deutlich und das eigene Schwanken in Bezug auf vollständige Wiedergabe angedeutet bleibt; vgl. ders., „Neueste Mitteilungen über den Nachlaß von Karl Marx und Friedrich Engels", in: Archiv usw., Jg. 11 (1925), S. 385-400, bes. S. 396. D. Rjazanov, „Einleitung zum ersten Band des Briefwechsels zwischen Marx und Engels”, in: K. Marx, F. Engels, Historisch-kritische Gesamtausgabe, Abt. III, Bd 1, Berlin 1929, S. IX-L, plaziert durch ausführliche inhaltliche Polemik gegen die Tilgungskriterien des verantwortlichen Herausgebers die Schuldzuweisung für die Geschichtskosmetik von 1913 sehr einseitig bei Bernstein.

19 Nach Struik, ,Introduction”, a.a.O., S. 3f., begann Rjazanov seine politische Tätigkeit als Fünfzehnjähriger bei den Narodniki; vgl. auch $\mathrm{Nr} 7$. 
Anforderungen die Ausgabe genügen sollte, ist nur knapp angedeutet, dadurch wird umso deutlicher, daß sie nach Vollständigkeit, textkritischer Zuverlässigkeit, Kommentierung und Erschließung des Inhalts akademischen Editionen der Zeit jedenfalls nicht nachstehen sollte. Die Aufnahme unveröffentlichter Manuskripte - nicht ausdrücklich genannt, aber als Problem seit 1902 bekannt - dürfte unter dem Gesichtspunkt der Vollständigkeit als selbstverständlich erschienen sein. ${ }^{20} \mathrm{Ob}$ Briefe mitberücksichtigt werden sollten, bleibt offen, ist aber wahrscheinlich; daß man sich bei Kenntnis der Sachlage auch auf Marxens Exzerpte eingelassen hätte, ist mehr als zweifelhaft. Aber dieser Mangel an editorischer Opulenz sollte Heutige nicht darüber hinwegtäuschen, daß der Wiener Editionsplan gegenüber bisheriger Publikationspraxis ganz nachhaltig den Akzent von der Popularisierung zur Wissenschaft verschiebt.

Was der Sozialdemokratischen Partei Deutschlands in organisatorischer Hinsicht zugemutet wurde, war kaum weniger originell - sie sollte den Wiener Editionsplan als programmatisches Dokument und Arbeitsplan übernehmen. Natürlich waren demokratische Formen zu wahren. Der Vorstand würde seine Vorstellungen zur Marx-Edition, oder in Ermangelung zusammenhängender Planungen eben den Wiener Editionsplan, bekannt machen und in der Partei zur Diskussion stellen. Die Resultate würden in einem langfristigen Editionsprogramm zusammengefaßt, das vom Vorstand oder sogar vom Parteitag zu beschließen wäre. Mit der Durchführung des Programms würde der Vorstand ein Herausgeberkomitee betrauen, dem Kooptationsrecht zustünde. Mit Bebel war immerhin einer der beiden Parteivorsitzenden als dessen Mitglied vorgesehen.

Sowohl dieser Prozedurvorschlag für eine vom Parteivorstand initiierte Programmdiskussion über die Marx-Edition, als auch Brauns Idee, die selbe Diskussion in schärferer Gangart durch einen unter Marxisten abgestimmten Schritt an die Öffentlichkeit zu eröffnen, deuten an, daß man in Wien durchaus sah, daß der Vorschlag, sich für ein ,rein wissenschaftlich" konzipiertes Großprojekt wie die Marx-Gesamtausgabe als Organisation zu engagieren, die SPD auf neues Terrain drängte. ${ }^{21}$ Verbindliche Festlegungen waren hier nur nach den in der Massenpartei geltenden

20 Vgl. F. Mehring, „Vorwort des Herausgebers”, in: Aus dem literarischen Nachlaß von Karl Marx, Friedrich Engels und Ferdinand Lassalle, hrsg. von F. Mehring, 4 Bde, Stuttgart 1902, Bd 1, S. vII: verglichen mit dem zu Lebzeiten veröffentlichten hielt Mehring das ihm bekannte unveröffentlichte Material in den Nachlässen von Marx und Engels zwar nur für „,eine nicht bedeutsame Ergänzung”, die aber „unzweifelhaft in eine Gesamtausgabe ihrer Schriften" gehöre.

${ }^{21}$ F. Lassalle's Reden und Schriften. Neue Gesammtausgabe, hrsg. von E. Bernstein, 3 Bde, Berlin 1892-93, konnte ihrer Anlage nach trotz ihres parteioffiziellen Charakters nicht als Präzedenzfall dienen. 
Regeln der Meinungsbildung und Beschlußfassung, wenn möglich auf höchster Ebene, zu erreichen.

Die Dringlichkeit des Editionsplan ist ganz praktisch begründet. Nach damals geltendem Recht erloschen im deutschsprachigen Raum die letzten urheberrechtlichen Schutzfristen für zu Lebzeiten publizierte Werke von Marx mit Ablauf des dreißigsten Jahres nach dessen Tode; diese wurden am 1. Januar 1914, wie es im Editionsplan und anderswo euphemistisch heißt, "Gemeingut". ${ }^{22}$ Die Sorge der Wiener Planer galt aber weniger dieser virtuellen Sozialisierung, als vielmehr der dadurch geschaffenen Möglichkeit der individuellen Aneignung im freien verlegerischen Zugriff. Ein öffentlich und verbindlich angemeldeter Anspruch der SPD, die Marx-Edition selbst in die Hand zu nehmen, sollte diese Möglichkeit nach zwei Richtungen blockieren oder doch einschränken.

Argumentativ wurde der Zugriff bürgerlicher Verlage vorangestellt. Zum Exempel diente ein Fall, in dem eine editorische Absurdität aus dem Kröner-Verlag mit einem Qualitätsprodukt aus dem Hause Vorwärts konkurrierte ( $\mathrm{Nr}$ 1). Daneben klingt echte Sorge vor der Risikofreudigkeit im bürgerlichen Verlagswesen; eine dort gestartete Gesamtausgabe des Marxschen Werks würde spätere sozialdemokratische Aktivitäten vollends unmöglich machen ( $\mathrm{Nr}$ 6). Ein sozialdemokratischer Anspruch auf den eigenen Markt konnte solche Freude am Risiko effektiv mindern. Daneben stand anscheinend die größere Sorge, das Sein sozialdemokratischer Druckereibesitzer könne deren Bewußtsein als revisionistische Verleger so weit bestimmen, daß man ,sogar vielleicht in München”, d.h. in Georg von Vollmars Hausverlag Birk \& Co., Marx drucken würde (Nr 5). Da die sozialdemokratischen Regionalverlage in ihrer Programmgestaltung weitgehend autonom waren, war marxpublizistischer Wildwuchs in den eigenen Reihen keineswegs ausgeschlossen. Eine programmatische Entscheidung der Partei konnte dem vorbeugen. Damit ergab sich ein knapper Terminplan. Die gewünschten Wirkungen waren nur zu erzielen, wenn ein dem Wiener Vorschlag entsprechendes Marx-Editionsprogramm der SPD rechtzeitig vor Ende 1913 beschlossen, besser noch in Angriff genommen wurde.

Die wissenschaftliche Gesamtausgabe der Werke von Marx als Mittelpunkt eines dringlichen Editionsprogramms der Sozialdemokratischen Partei Deutschlands ist das Novum des Wiener Editionsplans. Die personellen Vorschläge für das Herausgeberkomitee stellen über Schlüsselfi- 
guren der damaligen Marx-Forschung und -Edition die Verbindung zur bisherigen sozialdemokratischen Editionspraxis her.

Bebel war buchstäblich Schlüssel-Figur als literarischer Erbe von Engels. ${ }^{23}$ Dessen Nachlaß war für die Marx-Ausgabe, vollends wenn man sie mit derjenigen der Werke von Engels verbinden wollte, unentbehrlich. Bebel konnte den Zugang auch gegen Widerstand des Miterben Bernstein ermöglichen. ${ }^{24}$ Bebel war nach dem Tode von Marx einer der ersten gewesen, die die für Freund und Gegner selbstverständliche Erwartung, Engels werde das im Vorwort zum ersten Band des Kapital formulierte wissenschaftliche Programm einlösen, diesem vorgetragen hatten. ${ }^{25}$ Gleichzeitig stand er dem Wunsch nahe, den Toten nicht nur mit einem „Denkstein seitens der Gesamtpartei” oder der „Sozialisten aller Länder”, sondern auch durch eine „Volksausgabe seiner sämtlichen Schriften” zu ehren. ${ }^{26}$ Engels hatte sich gegen diese eher monumentale Pietät und für die Edition der Marxschen Kapital-Manuskripte entschieden. Sollte Nachfolge notwendig werden, hatte er schon 1889 Kautsky und Bernstein vorgesehen. ${ }^{27}$ Kautsky hatte das Editionsprogramm weitergeführt und im Jahre 1910 mit dem dritten Band der Theorien über den Mehrwert gerade abgeschlossen. ${ }^{28}$ Mehring hatte in seiner Ausgabe Aus dem literarischen

23 Engels vermachte seinen Manuskript- und Briefnachlaß, soweit er nicht als von Marx stammend dessen Töchtern hinterlassen oder den Briefpartnern zurückgegeben wurde, an Bebel und Bernstein, das Legat umfaßte ausdrücklich Marxens Briefe an Engels. Es nahm unter den im engeren Sinne politischen Legaten insofern eine Sonderstellung ein, als in allen anderen Fällen zugunsten der beiden Parteivorsitzenden Bebel und Singer verfügt worden war. Vgl. die deutschen Übersetzungen der einschlägigen Verfügungen, [„,Engels Testament vom 29. Juli 1893”] und [„Nachtrag zu Engels' Testament 26. Juli 1895"], in: K. Marx, F. Engels, Werke, Berlin 1956-68 (weiter MEW), Bd 39, S. 505-11.

24 So Brauns Erfahrung, als er diesen Zugang für die Bearbeitung des Urkundenbuches suchte; vgl. A. Braun an K. Kautsky, 20.3.1909, Nachlaß Kautsky D VI 295.

25 A. Bebel and F. Engels, 17.3.1883, in August Bebels Briefwechsel mit Friedrich Engels, hrsg. von W. Blumenberg (Quellen und Untersuchungen zur Geschichte der deutschen und österreichischen Arbeiterbewegung, Bd 6), Den Haag usw. 1965, S. 15If; vgl. auch die Schreiben vom gleichen Datum von F. Domela Nieuwenhuis und A. Loria, sowie P. L. Lavrov an Engels, 26.3., Marx-Engels-Nachlaß L 5105, 3647, sowie 3302, IISG.

${ }^{26}$ Bebel an Engels, a.a.O., und dessen Antwort vom 30.4., ebd., S. 152f.; vgl. auch den fast gleichzeitigen analogen Vorschlag russischer Sozialisten im Protokoll über den Kongreß der deutschen Sozialdemokratie in Kopenhagen [. . .]. Hottingen-Zürich 1883, S. 31 .

27 F. Engels an K. Kautsky, 28.1.1889, in Friedrich Engels' Briefwechsel mit Karl Kautsky, hrsg. von B. Kautsky (Quellen und Untersuchungen zur Geschichte der deutschen und österreichischen Arbeiterbewegung, Bd 1), Wien 1955 (weiter EngelsKautsky), S. 227-29.

28 Theorien über den Mehrwert. Aus dem nachgelassenen Manuskript „Zur Kritik der politischen Ökonomie" von Karl Marx, hrsg. von K. Kautsky, 3 Bde in 4 Tln, Stuttgart 1905-10. 
Nachlaß von 1902 an zwei andere Editionsvorhaben von Engels anknüpfen können. Dieser hatte sich eine Marx-Gesamtausgabe nachdrücklich vorbehalten. ${ }^{29}$ Eine „Gesamtausgabe dieser kleinen Arbeiten [. . .], sowohl der von M. wie von mir 1842-1852 erschienenen Artikel", beabsichtigte er dann in seinem letzten Lebensjahr und gewann Mehrings Unterstützung bei der Sammlung des verstreuten und unzugänglichen Materials. ${ }^{30}$ Der andere Plan, Lassalles Briefe an Marx und Engels zu veröffentlichen, entstand 1891 in Folge der Kontroversen um die Kritik des Gothaer Programms. Man hatte Engels die Verletzung Lassallescher Traditionen in der Sozialdemokratie vorgeworfen. Diesen Traditionen, die für ihn verzichtbar waren, sollte die Veröffentlichung der Lassalle-Briefe entgegenwirken, indem sie deren Autor in derjenigen historischen Größenordnung zeigte, die ihm nach Engels zukam. ${ }^{31}$ Mehrings Kombination dieser Briefe mit gesammelten Schriften der Adressaten drückte dann allerdings ein stark abweichendes Verständnis der Proportionalverhältnisse aus. ${ }^{32}$

Dietz war, wenn überhaupt über eine wissenschaftliche Gesamtausgabe geredet werden sollte, gleichsam die verlegerische Bedingung ihrer Möglichkeit. Die deutsche Ausgabe der Misère, übersetzt von Bernstein und Kautsky, kommentiert von Engels, hatte 1885 die Spezialisierung seines Verlagsprogramms auf die Verbreitung des wissenschaftlichen Sozialismus eingeleitet. ${ }^{33}$ Die 1910 in Deutschland greifbaren Marxiana, soweit sie nicht für täglichen Agitationsgebrauch bestimmt oder, wie die Bände des Kapital, noch anders vertraglich festgelegt waren, gehörten zu

29 Vgl. die Ablehnung des von Paul Ernst stammenden Planes, F. Engels an W. Liebknecht, 18.12.1890, MEW, Bd 37. S. 527, und deren Begründung, ders. an K. Kautsky, [29.6.1891,] Engels-Kautsky, S. 304f., MEW, Bd 38, S. 125f.

${ }^{30} \mathrm{Vgl}$. zu den späteren Plänen u.a. F. Engels an L. Lafargue, 17.12.1894, an L. Kugelmann, 1.1.1895, und an R. Fischer, 15.4., MEW, Bd 39, S. 347, 359, 467; zur Beteiligung von Mehring ders. an F. Mehring, [Ende April] und 9.5., ebd., S. $473 f$., 476.

${ }^{31}$ F. Engels an K. Kautsky, 23.2. und [29.6.]1891, Engels-Kautsky, S.282f., 304 (MEW, Bd 38, S. 40f., 125). Das Vorhaben war offensichtlich mit Bernsteins kritischer Einleitung zur parteioffiziellen Lassalle-Edition abgestimmt, vgl. ders. an L. Lafargue, 13.6.1891, MEW, Bd 38, S. 118.

32 Kautsky bezeichnete die Briefe privatim als Rehabilitierung Lassalles, an V. Adler, 21.11.1901, in: Adler-Briefwechsel, S. 381. Bernstein, dessen Darstellung von 1892 Mehring ausdrücklich revidieren wollte, betonte gegenüber dem ,freundlichen Eindruck” die „unfreundlichen Tatsachen”, vgl. E. Bernstein, „Über das Verhältnis von Lassalle zu Marx und Engels", in: Dokumente des Sozialismus, Bd 1 (1902), S. 157-77, hier S. 158.

33 K. Marx, Das Elend der Philosophie. Antwort auf Proudhons „Philosophie des Elends”. Deutsch von E. Bernstein und K. Kautsky, mit Vorwort und Noten von Friedrich Engels, Stuttgart 1885. Vgl. auch den Kommentar des Herausgebers der neubearbeiteten 11. Auflage in: Dass. Nach der deutschen Übers. von E. Bernstein [. . .] neu hrsg. [...] von H. Pelger, Bonn 1979, bes. S. IX-XIV. 
diesem Programm. ${ }^{34}$ Neben den genannten Editionen von Kautsky und Mehring befand sich darunter der Sorge-Briefwechsel mit seinem hohen Anteil an Briefen von Marx und Engels. ${ }^{35}$

Noch Rjazanovs laufende Arbeitsvorhaben lassen sich in den $\mathrm{Zu}$ sammenhang, der sich hier andeutet, einfügen. Das Urkundenbuch der Internationale hätte inhaltlich einer historiographischen Absicht von Engels, die dieser im Rahmen seiner Marx-Biographie realisieren wollte, entsprochen. ${ }^{36}$ Die Probleme bei der Übersetzung der Eastern Question ergaben sich direkt aus den Schwächen der Vorlage. Engels hatte die 1850-er Jahre nur bedingt zu den „Hauptkapitel[n] aus Mohrs politischem Leben gerechnet", hielt die Abläufe aus der öffentlichen Polemik für rekonstruierbar und Widerlegenswertes für durch Marx selbst widerlegt. An der Publizistik jener Jahre war er anscheinend weniger interessiert, zudem überzeugt, sie hinreichend verfügbar zu haben. ${ }^{37}$ Es ist mindestens zu erwägen, ob bei größerem Interesse seinerseits jene Schwächen teilweise vermeidbar gewesen wären. ${ }^{38}$

Wenn man sich die Edition von Marx-Texten bis 1910 in einem weitgehend durch inhaltliche, aber auch durch persönliche Vorentscheidungen, Pläne und Wünsche von Engels geprägten Zusammenhang vorstellt, trifft das auch das Zeitverständnis - noch die Herausgabe des Briefwechsels zwischen Marx und Engels wurde 1913 mit dem Wunsch des Letzteren legitimiert. ${ }^{39}$ Wer zu Jahresende 1910 sehr optimistisch bilanzierte, hätte sogar sagen können, ein großer Teil jener Pläne und Wünsche sei inzwi-

$34 \mathrm{Vgl}$. die Bibliographie des Verlages in B. Emig. M. Schwarz, R. Zimmermann, Literatur für eine neue Wirklichkeit [. . .], Bonn usw. 1981. bes. S. 35-79.

${ }^{35}$ Briefe und Auszüge aus Briefen von Joh. Phil. Becker [. . .] an F. A. Sorge und Andere, Stuttgart 1906.

36 Vgl. z.B. F. Engels an L. Lafargue, 24.6.1883, MEW, Bd 36, S. 43, und ders. an K. Kautsky, 25.3.1895, Engels-Kautsky, S. 427 und die Folgekorrespondenz.

37 Vgl. zur Frage der „Hauptkapitel aus Mohrs politischen Leben” F. Engels an L. Lafargue, 17.12.1894, MEW, Bd 39, S. 347; dazu und zur apologetischen Erledigung der Emigrationszeit bis 1860 durch Marx selbst ders. an Kautsky, a.a.O.; zur Frage der amerikanischen Publizistik die Erwähnungen der Sammlung Meyer, die später als Grundlage für die englischen Veröffentlichungen diente, in den Briefen des Jahres 1895 , MEW, Bd 39, S. 359, 375, 397 und Anm. 385.

38 Daß daneben die Trennung der inhaltlich eng verbundenen Nachlässe von Marx und Engels, insbesondere bei der Fehlzuschreibung der Artikel über Revolution und Konterrevolution in Deutschland, wie Kautsky es sieht, eine wichtige, wenn nicht entscheidende Rolle spielte, wird nicht bestritten. Vgl. K. Kautsky, „Engels' Nachlaß”, in: Engels-Kautsky, S. 445-57, hier S. 447.

39 Indirekt durch Berufung auf die testamentarische Verfügung über den Briefwechsel bei A. Bebel, E. Bernstein, ,Vorwort”, in: Der Briefwechsel zwischen Engels und Marx, a.a.O., S. [v], direkt Mehring, ,Engels und Marx”, a.a.O., S. 1; zum Wunsch von Engels vgl. Bebel an Adler, 22.3.1911, a.a.O., S. 528. 
schen realisiert oder befinde sich mit Aussicht auf baldigen Abschluß in Arbeit. Offen sei die Gesamtausgabe, sie werde über das, was Engels im Auge gehabt habe, wesentlich hinausgehen, sei aber als Desiderat von Mehring 1902 ausdrücklich anerkannt. ${ }^{40}$ Von den Vorarbeiten, die 1902 noch fehlten, sei mit der Erfassung der publizistischen Arbeiten der 1850er Jahre eine der schwierigsten inzwischen erfolgt. ${ }^{41}$ Was Mehring seinerzeit auf absehbare Zeit unmöglich erschien, sei inzwischen in den Bereich des Möglichen gerückt. So etwa hätte Rjazanov am 30.12.1910 in Wien referieren können. ${ }^{42}$ In einer solchen Bilanz wären allerdings die politischen und persönlichen Probleme, die diesem Zusammenhang inzwischen eigen waren, ausgeklammert geblieben. Ausgeklammert wie der Name Bernstein im Vorschlag für das Herausgeberkomitee, wo er natürlich nicht zufällig fehlt. Kautskys Leitung und die Besetzung des Komitees mit zuverlässigen Marxisten mußten sicherstellen, daß die Marx-Ausgabe nach den Vorstellungen jener bearbeitet wurde, ,die von der Bedeutung von Marxens Werken für den Befreiungskampf des Proletariats überzeugt sind" (Nr 1).

Bebel hatte 1899 auf dem Parteitag von Hannover kategorisch erklärt, $\mathrm{da} ß$ er Bernstein nach dessen Revision des Marxismus für früher verabredete editorische und biographische Arbeiten über Marx und Engels für „vollkommen unqualifiziert” halte, und dessen Stellung als Testamentsvollstrecker und literarischer Erbe von Engels faktisch zur Diskussion gestellt. ${ }^{43}$ Laura Lafargue bemühte sich seit 1901 bei Bebel um die

40 Vgl. unten, S. 118.

$41 \mathrm{Vgl}$, auch die Reaktion von Dietz auf die Londoner Funde von Rjazanov und deren Konsequenzen für die „Übersetzung” der Eastern Question: „,Da doch einmal die Werke Marx', wozu auch seine Zeitungsartikel, Korrespondenzen gehören, veröffentlicht werden müssen, so glaube ich, daß eine umfassende Berücksichtigung in Goldendachs und Ihrem Sinne zweckmäßig ist. Es sollte mich freuen, wenn ich zu diesen Publikationen als Verleger beitragen kann. Damit ist für die Literatur u. das nachwachsende Geschlecht keine unerhebliche Arbeit geleistet." H. Dietz an K. Kautsky, 31.10.1909, Nachlaß Kautsky D VIII 436.

${ }^{42} \mathrm{Vgl}$. dazu die in der Beurteilung des Geleisteten skeptischere Bilanz, die Rjazanovs kritischem Verhältnis zu den Arbeiten seiner Vorgänger besser entspricht, trotzdem auf die ,wissenschaftliche Ausgabe aller Werke von Marx und Engels" zugespitzt ist: Rjasanoff, ,Der Briefwechsel zwischen Marx und Engels”, a.a.O., S. 565-67.

${ }^{43}$ Bebel sprach laut Vorwärts-Bericht von „Bearbeitung des Kapital” und „MarxBiographie", Laura Lafargue notierte über die literarischen Testamentsvollstrecker von Engels und deren Verabredungen bissig, ,[they] are not called to decide in the matter." Was weitergegeben wurde: laut Buchprotokoll sprach Bebel über die Bearbeitung des literarischen Nachlasses von Engels und dessen Biographie. Vgl. den Verhandlungsbericht im Vorwärts, Jg. 16, Nr 241 (14.10.1899), 1. Beilage, S. [3]; L. Lafargue an K. Kautsky, 19.10.1899, Nachlaß Kautsky D XV 13; Protokoll über die Verhandlungen des Parteitages [. . .] zu Hannover [. . .], Berlin 1899, S. 229. 
Rückgabe der Briefe ihres Vaters an Engels. Die nachträgliche Revision der entsprechenden testamentarischen Verfügung von Engels sollte revisionistischem Mißbrauch der Briefe durch Bernstein vorbeugen. Dieser Versuch scheiterte allerdings, trotz anfänglicher Zusagen Bebels, an dessen definitiver Ablehnung im Jahre 1909. Ihre Verstimmung war groß, für einen Moment erwog sie sogar juristische Schritte. ${ }^{44}$

Kautsky berief sich 1905 darauf, daß Engels ihn als denjenigen designiert habe, der ihn im Todesfall bei der Herausgabe des IV. Bandes des Kapital ersetzen könne, stieß aber auf berechtigten Widerspruch Bernsteins. ${ }^{45}$ Die folgende erhitzte Polemik darüber, ob Engels einen oder zwei Nachfolge-Editoren bezeichnet habe, fügte dem bisherigen eine neue Dimension hinzu. ${ }^{46}$ Bisher hatte Bernsteins gegenwärtige wissenschaftliche, politische und moralische Qualifikation, auch die Frage, ob er angesichts seiner Wandlung überhaupt noch als Erbe von Engels auftreten könne, zur Debatte gestanden. Jetzt wurde die Legitimitätsfrage gleichsam rückwirkend gestellt, denn man stritt um Vorgänge des Jahres 1889.47

Braun versuchte 1909 für die Bearbeitung des späteren Urkundenbuches den Zugang zum Engels-Nachlaß zu sichern. Die zögernde Reaktion Bernsteins führte zu der Vermutung, dieser suche Mitsprache bei der Wahl des Bearbeiters oder reflektiere sogar selbst. Die Abwehr Brauns, der sich „eher einen rein objektiven bürgerlichen Gelehrten, als Ede" als Bearbeiter vorstellen konnte, ${ }^{48}$ verschärft die skizzierte Tendenz in der Formulierung der Alternative.

Die Absicht des Wiener Editionsplans, die Marx-Gesamtausgabe zur Angelegenheit der Marxisten in der Sozialdemokratie zu machen, setzt inhaltlich die bisherige Praxis fort. Politische, privatrechtliche und persönliche Auffassungen, Ansprüche und Verdienste, die für den $\mathrm{Zu}$ sammenhang dieser bisherigen Praxis charakteristisch waren, werden in der Zusammensetzung des Herausgeberkomitees berücksichtigt, soweit sie dieser Absicht nicht im Wege stehen, und damit in den neuen, formell

${ }^{44}$ Vgl. über die Bemühungen bis 1909 zusammenfassend L. Lafargue an K. Kautsky, 12. und 21.6.1909; über Bebels Ablehnung und eventuelle juristische Schritte, ebenso, 1.9.1909; über eine für sie negative Auskunft vom früheren Anwalt von Engels, Crosse, ebenso, 12.1.1910. Nachlaß Kautsky D XV 45, 46, 48, 51. Weiter s. unten, S. 119f.

${ }^{45}$ Theorien über den Mehrwert, a.a.O., Bd 1, S. vIII, dann Bernsteins Rezension der Bände 1 und 2 in: Dokumente des Sozialismus, Bd 5 (1905), S. 445.

${ }^{46}$ Die Polemik mit Úberschneidungen und wenigen wechselseitigen Ergänzungen in der Neuen Zeit, Jg. 24 (1905-06), Bd 1, S. 167f., 303f., 374-76, 470-72, und in Dokumente des Sozialismus, Bd 5, S. 527f., 566-68.

$47 \mathrm{Vgl}$. Anm. 27; Kautsky hat die für seine Argumentation zentrale Behauptung, Engels habe ihn schon vor 1889 designiert, im Alter faktisch fallen gelassen, vgl. seine Kommentare in Engels-Kautsky, S. 229, 446, 450.

48 Braun an Kautsky, 20.3.1909, a.a.O. 
organisierten Rahmen übernommen. Im gegenteiligen Falle, und derjenige von Bernstein ist nur der politische und privatrechtliche Extremfall, nahm man anscheinend an, daß die Legitimation des Herausgeberkomitees durch Parteibeschluß derjenigen der persönlich geprägten älteren Ansprüche übergeordnet sei und sie neutralisieren könne.

Der Wiener Editionsplan lag Anfang Januar 1911 zunächst Kautsky, dann anderen für das Herausgeberkomitee in's Auge gefaßten deutschen Kandidaten vor. Deren Zustimmung mußte über sein weiteres Schicksal entscheiden, denn mit einem innermarxistischen Streit konnte man die öffentliche Diskussion über ein Marx-Editionsprogramm der Partei nicht beginnen, ohne sein Ziel von vornherein zu verfehlen. Reaktionen von Dietz und Mehring sind direkt überliefert, diejenigen von Kautsky lassen sich aus den Antworten von Braun und Rjazanov rekonstruieren. Demnach hätte sich schon um die Monatsmitte ein Konsens der Ablehnung des Planes auf verschiedenen Ebenen herausgebildet.

Der Dringlichkeit, die durch das Urheberrecht gegeben war, standen Dietz, Kautsky und Mehring wesentlich gelassener gegenüber als Braun. Mit dem Vorstandsbeschluß, bis 1914 die Volksausgabe des ersten Bandes des Kapital vorzubereiten, war das „zunächst Notwendige” wohl auch getan. Für die folgenden Bände des Werkes galten aufgrund der editorischen Bearbeitung durch Engels und Kautsky andere Schutzfristen, das unpublizierte Oeuvre befand sich in der Verfügung der Erben und damit immerhin in Reichweite der Partei.

Außerdem ist die Volksausgabe im Gespräch mit Wien als taktisches Instrument eingesetzt worden: Ecksteins Schweigen darüber, dann Dietzens Bitte um offiziöse Mitteilung $(\mathrm{Nr} 3)$ und schlieBlich dessen schneller Sinneswandel über die Teilnahme Rjazanovs an der Bearbeitung zeigen das. ${ }^{49}$ Dieser - im Kreise der Wiener Planer derjenige, der sich am stärksten mit Editionsarbeiten beschäftigte - wurde in ein dringliches parteioffizielles Projekt gezogen, das ihn, wie seine Reaktion zeigt, inhaltlich, vor allem auch von seinem politischen Gebrauchswert her, außerordentlich interessierte $(\mathrm{Nr} 7)$.

Die Frage, ob der Plan realisierbar und in der Partei durchzusetzen sei, wurde mit unterschiedlichen Gründen übereinstimmend negativ beantwortet. Die Weigerung von Dietz, sich auf Wiener Projekte einzulassen, spricht für sich. Sie war durch die Annahme, sein Manuskriptschuldner

49 Auf den Vorschlag von Kautsky, Rjazanov zu beteiligen, reagierte Dietz in erster Instanz außerordentlich zurückhaltend, am 17.2.1911 war. der Vertrag geschlossen; an K. Kautsky, 8.1.1911, und Rjazanov an K. Kautsky, 17.2.1911, Nachlaß Kautsky D VIII 459 und D XIX 326. 
Rjazanov sei der Urheber des Plans, sicher mitbestimmt ( $\mathrm{Nr}$ 3). Die Erwähnung von Richard Fischer gehört aber in den gleichen Kontext und deutet an, daß auch Widerstand aus dem Vorwärts-Verlag zumindest in's Gespräch gebracht wurde ( $\mathrm{Nr} 7$ ). Mehring sah keinen Anlaß, seine im Forschungsstand begründete Auffassung von 1902 zu ändern. Diese liest sich als Kommentar zu seinen lakonischen Bemerkungen von 1911:

Eine wissenschaftliche Gesamtausgabe der Schriften, die Karl Marx und Friedrich Engels hinterlassen haben, wäre eine so wünschenswerte Sache, wie sie in absehbarer Zeit eine unmögliche Sache ist. Für ihre würdige Herstellung ist noch eine Reihe von Vorarbeiten notwendig, die von keinem Einzelnen, und selbst von Mehreren nicht binnen kurzer Frist erledigt werden können. ${ }^{50}$

Kautsky hat zunächst vielleicht geschwankt ( $\mathrm{Nr} 3$ ), er detaillierte seine Einwände dann am weitesten. Er hatte gute, gesundheitliche Gründe, sich jeder Mehrbelastung zu entziehen. ${ }^{51}$ Die Frage Ecksteins, ob sich die Partei eine Gesamtausgabe personell-wissenschaftlich und finanziell leisten könne, hat er übernommen ( $\mathrm{Nr} 2$ und 6). Dazu kamen Zweifel daran, daß sich eine so umfangreiche und in ihren Konsequenzen schwer kalkulierbare wissenschaftliche Unternehmung mit den vorhandenen Mitteln organisatorisch planend bewältigen lasse $(\mathrm{Nr} 6)$. Schließlich wurde die Frage der politischen Durchsetzbarkeit in der Partei zumindest dort berührt, wo es um Eignung und Kriterien für die Auswahl der Bearbeiter für eine „Marx-Ausgabe der Marxisten” ging. Einzelne der hier vorgebrachten Argumente waren sicher plausibel und erleichterten Rjazanov den Entschluß, auf bessere Zeiten zu warten ( $\mathrm{Nr} 7$ ). Im Ganzen erscheinen sie auch dann vordergründig, wenn man die Überlieferung in Referat und Widerspruch des Gegenspielers berücksichtigt. Es fiel Braun leicht, diese Einwände in seiner Gegenargumentation nicht als unüberwindbare Hindernisse zu behandeln, sondern als vorhersehbare Schwierigkeiten, deren Überwindung die vorgeschlagene langfristige rationale Planung gerade ermöglichen sollte ( $\mathrm{Nr} 7$ ).

Eine dritte Ebene, auf der der organisatorische Grundgedanke des Wiener Vorschlags direkt berührt wurde, ist nur angedeutet. Kautsky hat Braun gegenüber die bisherige Praxis der vertraulichen, wenn nicht geheimen Publikationspläne verteidigt ( $\mathrm{Nr} 6$ ). Grundsätzlich ließ sich gegen den Vorschlag, ein Editionsprogramm der Partei in öffentlicher Diskussion und Beschlußfassung zu formulieren, kaum etwas einwenden. Viel weniger

50 Aus dem literarischen Nachlaß, Bd 1, S. [VII].

51 Vgl. G. P. Steenson, Karl Kautsky 1854-1938. Marxism in the classical years, Pittsburg 1978, S. 173. 
ließ sich die Ablehnung des Wiener Plans durch Hinweis auf die vorgeschlagene Prozedur vor der Parteiöffentlichkeit plausibel begründen. Dennoch war bei Bebel, Dietz, Kautsky und Mehring, die im Herausgeberkomitee Kontinuität zur bisherigen, von Engels begründeten und strukturierten Editionspraxis sichern sollten, möglicherweise gerade dieser Prozedurvorschlag aus praktischen Gründen für die Ablehnung entscheidend. Mit Bernstein, den der Wiener Plan ausschalten wollte, mit Laura Lafargue, die bei aller urheberrechtlichen Sensibilität dort nicht berücksichtigt worden war, und mit dem „Nachwuchsforscher" Rjazanov bildeten sie damals für den Marx-Engels-Briefwechsel ein Herausgeberkomitee ,.de facto", das der Tradition weit stärker verpflichtet war und dessen Tätigkeit durch öffentliche Diskussion durchkreuzt werden konnte.

Der Wunsch von Engels nach Publikation seines Briefwechsels mit Marx und seine testamentarische Verfügung, mit der er die Marx-Töchter überging und diesen Briefwechsel seinen literarischen Erben Bebel und Bernstein hinterließ, zwangen fünfzehn Jahre nach seinem Tode noch einmal einen Personenkreis zur Zusammenarbeit, der sich inzwischen weit, im Einzelfall bis zum unüberbrückbaren Gegensatz auseinanderentwickelt hatte..$^{52}$

Bebel hatte seine Disqualifikation Bernsteins als Herausgeber des Engels-Nachlasses zu revidieren. Bis Juni 1910 war dies geschehen; sobald die Briefausgabe in den Quellen Konturen annimmt, ist nur von Bernstein als Bearbeiter die Rede. Im Juli hatte der Parteivorstand sein Einverständnis mit dem Erscheinen der Ausgabe bei Dietz erklärt und einen Teil der Bearbeitungskosten übernommen. ${ }^{53}$ Laura Lafargue hatte eine solche Entwicklung seit längerem befürchtet. Ihre Bemühungen um Rückgabe der Marx-Briefe zielten 1909 ausdrücklich darauf, sie zu blockieren und die Benutzung und Veröffentlichung der Briefe durch Genossen ihres Vertrauens zu ermöglichen. ${ }^{54}$ Sie meinte, damit im Sinne von Engels zu handeln.

Si Engels vivait aujourd'hui, il arracherait cette correspondance aux griffes de ce faux bon-homme et faussaire et c'est parce qu'il n'est plus là que j'ai voulu le faire à sa place. ${ }^{55}$

Laura Lafargue hat ihr Urteil über Bernstein nicht revidiert, behielt sich

52 Soweit nicht anders nachgewiesen, ist der Fundort der in der folgenden Skizze erwähnten bzw. zitierten Briefe das ,Dossier Briefwechsel zur Ausgabe der Korrespondenz Marx-Engels". IISG; das Dossier stammt von H. Dietz, vgl. ders. an Adler, 29.5.1913, in: Adler-Briefwechsel, S. 569.

53 A. Bebel an H. Dietz, 15.7.1910.

${ }^{54}$ L. Lafargue an K. Kautsky, 21.6.1909, Nachlaß Kautsky D XV 46.

55 Ebenso, 12.6.1909, ebd. 45. 
auch das ihr urheberrechtlich zustehende Veto für den Fall des editorischen Mißbrauchs ausdrücklich vor. Die Zustimmung zur Publikation, um die sie Dietz im Juli 1910 ersuchte, hat sie dann aber, von Kautsky beraten, unter Bedingungen erteilt, die ihr Einsichtnahme und Mitsprache in allen Stadien der Bearbeitung sicherten. ${ }^{56}$ Für Bernstein war damit die Weiterführung der inzwischen schon relativ fortgeschrittenen Editionsarbeiten Anfang Juli schon hatte er Dietz die Briefe bis 1849 mit Erläuterungen und Vorwort druckfertig avisiert ${ }^{57}$ - gesichert. Mit einer durch geschärftes Mißtrauen geleiteten Mitsprache von Laura Lafargue hatte er sich abzufinden und war sich dessen bewußt: ,Sie scheint zu glauben, daß wenn man nicht mehr an die Transsubstantiationslehre glaubt, man sich sofort zum Banknotenfälscher entwickelt."58

Die Fragilität dieser Verabredungen zeigte sich, als die ersten Probeabzüge vorlagen. Rjazanov stand allein, als er nach erster Einsichtnahme die Vollständigkeit der Wiedergabe und Bernsteins Abstinenz in der Kommentierung begrüßte, er irrte in der Erwartung ,in dieser Gestalt wird die Arbeit keinen Anstoß finden bei den enragirtesten Anti Bernsteinianern." ${ }^{59}$ Bebel richtete seine Kritik zunächst auf Bernsteins Editionsarbeit: „Daß B. die Arbeit in so außerordentlicher dürftiger Weise mit Anmerkungen hinausgehen lassen würde, hätte ich nicht für möglich gehalten. Das ist mehr als leichtfertig gearbeitet, das ist liederlich." 60 Bei näherem Zusehen - und damit bleibt er nicht allein - erschütterten ihn zunehmend die Quellen selbst: „Warum die beiden Alten mit Vorliebe in den ordinären Ausdrücken schwelgen ist mir unfaßbar."61 Der Protest von Laura Lafargue fügte dem nur die praktische Folgerung hinzu: „A mon avis la publication in extenso de cette correspondance est inadmissible et des omissions s'imposent."62 Er komplizierte die Situation aber nachhaltig, da sich ihr bisheriger Berater Kautsky gleichzeitig krankheitshalber zurückziehen mußte ${ }^{63}$ und die Probeabzüge an den bis dahin wohl unbeteiligten

${ }^{56}$ L. Lafargue an H. Dietz, 20.7.1910; vgl. auch K. Kautsky an H. Dietz, 13. bzw. 15.7.1910, und L. Lafargue an K. Kautsky, 20.6 und 2.8.1910, letztere Nachlaß Kautsky D XV 52-53.

57 D.h. in der vierbändigen Ausgabe von 1913 ein Viertel des ersten Bandes; Bernsteins Zeitplan sah Anfang September Publikation des ersten von drei Bänden im November 1910, der folgenden zu Ostern bzw. Michaeli 1911 vor. Vgl. E. Bernstein an H. Dietz, 10.7. und 10.9.1910.

58 E. Bernstein an H. Dietz, 18.7.1910.

59 Rjazanov an H. Dietz, 10.9.1910.

60 A. Bebel an H. Dietz, 30.9.1910.

61 Ebenso, 6.10.1910.

62 L. Lafargue an H. Dietz, 7.10.1910.

${ }^{63}$ L. Kautsky an H. Dietz, 6.10.1910. 
Mehring weitergab. ${ }^{64}$ Dieser dramatisierte die stilistischen Einwände zu der Befürchtung, durch die Form des Briefwechsels würden

die zwanzigjährigen Anstrengungen, die Sie, Kautsky und ich gemacht haben, um das literarische Andenken der beiden Männer in würdiger Weise zu pflegen, mehr oder weniger illusorisch gemacht, kann auch der Partei der schwerste Schaden zugefügt werden, wenn eine halbwegs geschickte Feder der Gegner die Menschlichkeiten des Briefwechsels in einem Wahlpamphlet verarbeitet. ${ }^{65}$

Mehring ging weiter und stellte außer den harschen Urteilen von Marx und Engels über ihre (Zeit-)Genossen auch ganze inhaltliche Komplexe des Briefwechsels, wie die Londoner Emigrationskonflikte und die Bangya-Affäre, zur Diskussion. Im Urteil des nach eigener Meinung besten lebenden Kenners der Materie mußten

die ganzen Flüchtlingsbriefe kas[s]iert werden. Sachlich ist daran nicht das Geringste verloren, denn die sachlichen Gründe, aus denen Marx u. Engels mit den demokratischen Flüchtlingen aneinandergeraten, und in denen sie vollständig im Rechte waren, sind in diesen Briefen nirgends erkennbar, und in dem endlosen Klatsch, den sie enthalten, finde selbst ich mich nicht zurecht $[\ldots]^{66}$

Dietz hat Mehring davon abgehalten, seine Einwände als Rundschreiben zu verbreiten, aber er machte sich die Kritik ganz weitgehend zu eigen. ${ }^{67}$ Er war auch nicht unbeteiligt daran, daß Laura Lafargue Mehring im November $1910 \mathrm{zu}$ ihrem Vertrauensmann für die Briefwechseledition bestimmte ${ }^{68}$ er hatte ihr die Benennung eines so bevollmächtigten, für ihn direkt erreichbaren Gesprächspartners vorgeschlagen. ${ }^{69}$

Die Verwicklungen rund um den Marx-Engels-Briefwechsel bis zum November 1910 wirken wie eine Illustration ,auf erweiterter Stufenleiter” für Bebels ironische Prognose vom Juli, es könne das Gegenteil dessen eintreten, was Laura Lafargue befürchte: „Bernstein berichtet objektiv u. sie korrigirt subjektiv." "70 Derartige Frontwechsel blieben für die Realisierung dieser letzten ,klassischen” sozialdemokratischen Marx-Engels-Edi-

64 F. Mehring an H. Dietz, 15.10.1910.

65 Ebd.

${ }^{66}$ F. Mehring, ,Rundschreiben an die Genossen Bebel, Bernstein, Dietz, Goldendach, Kautsky u. Frau Lafargue", 20.10.1910, Konzept von Mehring und Reinschrift von anderer Hand; Wiedergabe nach der Reinschrift.

67 H. Dietz an F. Mehring, 21.10.1910.

68 L. Lafargue an H. Dietz, 11.11.1910.

69 H. Dietz an L. Lafargue, 18.10.1910; Mehring gegenüber hatte er den Gedanken schon am Vortage ventiliert, dieser ließ sich schnell interessieren; vgl. H. Dietz an F. Mehring. 17.10.1910, und Mehring an Dietz, 19. und 27.10.1910.

70 A. Bebel an H. Dietz, 29.7.1910. 
tion charakteristisch und bestätigten auch noch Bebels pessimistische $\mathrm{Zu}$ sammenfassung eines Gesprächs mit Dietz von Ende Oktober: „Wir haben dann auch wegen der Marx-Engels-Ausgabe verhandelt; da wird's ja noch allerlei zu überstehen geben."71

Verallgemeinert und hypothetisch auf den Wiener Editionsplan zugespitzt, läßt sich der Stand der Angelegenheit zu Beginn des Jahres 1911 so zusammenfassen.

Der Parteivorstand hatte die Briefwechselausgabe gutgeheißen, unterstützte sie und gab ihr damit einen offiziellen Charakter. Möglich geworden war sie aber nur durch eine Reihe von politischen Kompromissen, vertraglichen Absprachen, Verabredungen und persönlichen Zugeständnissen. Der erreichte Modus vivendi wäre wahrscheinlich schon durch eine Bekanntgabe des Editionsvorhabens gefährdet worden, einer öffentlichen Parteidiskussion hätte er sicher nicht standgehalten.

Die Wahrscheinlichkeit, daß sich die aus dem Testament von Engels und dem Urheberrecht abgeleiteten Ansprüche durch Parteitagsbeschluß politisch überspielen ließen, war gering. Der Plan einer Gesamtausgabe, in dem Bernstein ausgeschaltet wurde und Laura Lafargue unberücksichtigt blieb, bot wenig Aussicht auf Verwirklichung.

Mit dem Blick in die Intimsphäre des Briefwechsels war bei allen Beteiligten das mehr oder weniger stark ausgeprägte Bedürfnis entstanden, politische Risiken zu vermeiden und Marx und Engels gegen sich selbst in Schutz zu nehmen. Ob eine auf Vollständigkeit zielende wissenschaftliche Gesamtausgabe inhaltlich überhaupt möglich sei, wurde fraglich.

Für die Beteiligten war damit gegen den Vorschlag, zu einem Editionsprogramm der Partei in öffentlicher Diskussion und Beschlußfassung zu kommen, eine Reihe von praktischen Hinderungsgründen gegeben, deren Überwindung in absehbarer Zeit nicht zu erwarten war. Diese Gründe eigneten sich ihrerseits kaum für öffentliche Erörterung und dies könnte erklären, warum der Wiener Editionsplan in aller Stille im Vorfeld der Entscheidung beerdigt wurde. Seinen formellen Adressaten, den Parteivorstand, erreichte er mit an Sicherheit grenzender Wahrscheinlichkeit nie. Wahrscheinlich kam nicht einmal eine Besprechung im Kreise des präsumptiven Herausgeberkomitees, auf die Hilferding in Berlin drängte, zustande. Wenn Rjazanov den Plan im Sommer 1911 als Argument gegen die Absicht von Laura Lafargue gebrauchte, die Manuskripte ihres Vaters dem British Museum zu vermachen ( $\mathrm{Nr} 11$ ), geschah dies sicher nur noch im eigenen Namen.

71 A. Bebel an L. Kautsky, 1.11.1910, a.a.O. (s. Anm. 1.) 
Es bleibt die Frage nach der Rolle Rjazanovs in der Konstellation zwischen Briefwechsel-Ausgabe und Wiener Editionsplan. Er war an den Vorbereitungen zur Ausgabe beteiligt und hatte Einblick in deren Kompliziertheit. Er unterzeichnete den Plan und war, auch wenn Braun als Hauptverantwortlicher angenommen werden muß, sachverständiger Mitautor. Hat er seine Wiener Freunde in Kenntnis jener Hindernisse zu einem aussichtslosen Vorstoß ermutigt?

Es war ein Vertrauensbeweis, daß er Einsicht in Bernsteins Editionsarbeiten erhielt. ${ }^{72}$ Er blieb aber Neuling und relativer Außenseiter, es ist zu bezweifeln, daß er über alle Einzelheiten informiert war. ${ }^{73} \mathrm{Er}$ hat darum die Stabilität der gefundenen Lösungen möglicherweise überschätzt. Das Engagement des Parteivorstandes an der Briefwechseledition konnte zu der Erwartung führen, in anderem Kontext sei es möglich, die rechtlichen Ansprüche politisch abzulösen, im Falle von Laura Lafargue konnte man daran denken, daß Mehring auch im Herausgeberkomitee als ihr Vertrauensmann auftreten werde. Bedenken gegen den Inhalt der Briefe hat Rjazanov bald geteilt.

Die Briefe sind selbst für eine solche Archivratte, wie ich, manchmal schrecklich langweilig. Mehr als die Hälfte kann man ruhig liegen lassen, bis die große Ausgabe der gesammelten Werke von M[arx] und Eng[els] veröffentlicht wird. Und jetzt muß man sehr vieles streichen. Sonst gibt es einen großen Skandal. ${ }^{74}$

Er reagierte aber anders, als seinen älteren Kollegen hier unterstellt wird. Das resultierte aus der Praxis seiner Editionsarbeiten. Bei der Bearbeitung der Publizistik der 1850-er Jahre hatte er sich über die Qual der Auswahl aus den eigenen Forschungsergebnissen mit dem Blick auf die zukünftige Gesamtausgabe getröstet; diese war abstraktes Desiderat, höchstens konventionell an den Jubiläumsjahren 1918 und 1920 orientiert. ${ }^{75}$ Die gleiche Aussicht erleichterte es ihm offensichtlich, im Herbst 1910 bei der

72 Zweck war in erster Linie eine geplante (Auswahl-)Übersetzung der Briefe in's Russische: Rjazanov bemühte sich um Zustimmung zur allgemeinen Benutzung für seine Forschungsarbeiten an den Gesammelten Schriften und am Urkundenbuch: vgl. den Entwurf eines Verlagsvertrages zwischen Dietz und Bernstein vom 9.10.1910 und Rjazanov an Dietz, 3.11.1910.

73 Über Bernsteins Zeitplan z.B. war er unangenehm überrascht; vgl. Rjazanov an Dietz, 10.9.1910.

${ }^{74}$ Rjazanov an L. Kautsky. [vor dem 2.12.1910,] Nachlaß Kautsky D XIX 313.

75 Rjazanov an H. Dietz, 10.9.1910: „Man kann jetzt in die Ausgabe des Nachlasses der 50 und 60er Jahre nur das literarisch und wissenschaftlich am besten bearbeitetes [!] Material aufnehmen, und den Rest für eine künftige Ausgabe der Sammelwerke aufzubewahren [!]. - Sagen wir bis zum 1918-1920, das hundertjährige Jubiläum Marx und Engels." 
Briefwechselausgabe für Kürzung und Kosmetik am Geschichtsbild zu optieren. Ein Plan, der sich am dreißigsten Todestag von Marx und dessen rechtlichen Konsequenzen orientierte, bot die Möglichkeit, sich dem Desiderat in konkreten Schritten zu nähern.

Mit seiner Beteiligung am Wiener Editionsplan beruhigte sich Rjazanov im Widerspruch. Die gleichen Texte oder Textpassagen, die in der Briefwechselausgabe als skandalträchtig zu unterdrücken waren, sollten in einer beschleunigten Gesamtausgabe nur wenig später risikolos erscheinen können? ${ }^{76}$ Formal fügt sich diese Unterscheidung jener zwischen wissenschaftlicher Edition und popularisierender Massenverbreitung, die der Editionsplan vornimmt. Aber inhaltlich scheint neben dem Unterschied in der politischen Zielrichtung der Gedanke mitzuspielen, die wissenschaftliche Edition sei im Vergleich zur populären Ausgabe in gewisser Hinsicht politisch neutral in ihrer Wirkung.

Damit wäre eine Mittelstellung Rjazanovs zwischen den Wiener Marxisten und ihren Wunschpartnern in Deutschland gekennzeichnet. Die politischen Erwartungen, die hier wie dort mit einer wissenschaftlichen Gesamtausgabe der Werke von Marx verbunden wurden, waren, wenn nicht gegensätzlich, so doch mindestens sehr unterschiedlich. In Wien rechnete man die Edition wohl zu den bisher nicht beschrittenen Forschungsfeldern, deren sich die marxistische Wissenschaft zu bemächtigen hatte, um sie für die sozialistische Politik nutzbar zu machen. Der Übergang von der popularisierenden Verbreitung zur wissenschaftlichen Edition wäre auf diesem Feld jene ,,bewußte Verknüpfung der marxistischen Denk resultate und -Methoden mit dem gesamten modernen Geistesleben" gewesen, mit der der Marxismus dogmatische Erstarrung vermeiden und seinen wissenschaftlichen Anspruch einlösen konnte. ${ }^{77}$ In der Überzeugung, daß sich die „Bedeutung von Marxens Werken für den Emanzipationskampf des Proletariats" auch und gerade in der Form der Gesamtausgabe auswirken werde, konnte Braun sie der Partei als „Gegenwartsaufgabe" antragen.

War, wie in Berlin, diese Überzeugung weniger stark, fiel der Blick zuerst auf die ornamentalen Aspekte der akademisch-konventionellen Form. Eckstein stellte die Frage, ob sich die Partei den Luxus leisten

76 Von verändertem Standpunkt vermeidet 1914 das Plädoyer ex-post für die ungekürzte Publikation des Briefwechsels diesen Widerspruch und nimmt unfreundliche Interpretation und Ausschlachtung durch politische Gegner als unvermeidlich bewußt in Kauf; vgl. Rjasanoff, ,.Der Briefwechsel zwischen Marx und Engels”, a.a.O., S. 568.

it Marx-Studien, Bd 1, S. vilf. 
könne, mit einer wissenschaftlichen Gesamtausgabe überwiegend „dekorative Zwecke" zu verfolgen. Hinzu kam die Möglichkeit, daß der Luxus mit politischen Risiken verbunden war. Kautsky hatte die Frage anscheinend eindeutig beantwortet und mit den „Privatdozenten einer künftigen sozialistischen Universität" ironisch Herausgeber benannt, deren Arbeitsplatz doch wohl recht weit hinten am ,Weg zur Macht” gedacht werden mußte.

Am dreißigsten Todestag von Marx war ein Editionsprogramm der SPD noch nicht beschlossen worden, noch viel weniger wurden die Arbeiten an einer Marx-Gesamtausgabe schutzfristgerecht vor Ende 1913 aufgenommen. Der Wiener Editionsplan blieb Episode.

Der Wiener Editionsplan ( $\mathrm{Nr}$ l) befindet sich als Durchschlag mit handschriftlichen Korrekturen von Adolf Braun in einem Dossier mit Einzelstücken unterschiedlichen Charakters, die die Arbeit des ehemaligen Archivs der SPD in Berlin betreffen. Das Dossier wurde im IISG zusammengestellt, die Provenienz der jeweiligen Stücke ist im Einzelfall nicht mit letzter Sicherheit zu bestimmen, dürfte aber im Allgemeinen dem Sachbetreff entsprechen. Für den Durchschlag des Editionsplans ist anzunehmen, daß ihn Braun selbst, der in den 1920-er Jahren im Vorstand der SPD u.a. für die Arbeit des Parteiarchivs zuständig war, dort deponierte.

Im Nachlaß von Karl Kautsky und im Familienarchiv Kautsky fanden sich eine Reihe von Briefen der Beteiligten, die den Plan ganz oder teilweise direkt betreffen ( $\mathrm{Nr} 2-11$ ). Diese Briefe werden vollständig oder in ihren einschlägigen Teilen als Auszug wiedergegeben. Wo, wie in den Briefen von Rjazanov, Zweifel darüber bestehen könnte, ob Briefpassagen das Thema der Dokumentation berühren oder nicht, ist positiv entschieden und die Grenze weit gezogen worden. Über den Inhalt der weggelassenen Briefstellen orientieren knappe Regesten.

Die Wiedergabe der maschinenschriftlichen Texte erfolgt in der Fassung nach der handschriftlichen Korrektur. Diese Korrekturen, ebenso Korrekturen, Einfügungen usw. in den handschriftlichen Briefen, werden im Einzelnen nicht nachgewiesen. Flüchtigkeitsfehler wurden stillschweigend berichtigt, Orthographie und Zeichensetzung sind nach heutigen Regeln vereinheitlicht, die bei Mehring häufigen Verkürzungen und Verschleifungen wurden aufgelöst. Beibehalten wurden die orthographischen Schwankungen in den Briefen Rjazanovs, hier hätten Eingriffe den Briefcharakter verändert. Die Schreibungen ,Komite” ( $\mathrm{Nr} 1)$ und ,wol” ( $\mathrm{Nr} 2)$ deuten auf spezifische orthographische Gewohnheiten und wurden ebenfalls beibehalten. 
Die Werke von Karl Marx nach Erlöschen des Urheberschutzes. ${ }^{a}$

Am 14. März 1913 sind dreißig Jahre verflossen, seitdem Karl Marx aus dem Leben geschieden ist. Mit diesem Tage werden auch im deutschen Sprachgebiete seine Werke Gemeingut. Es ist anzunehmen, daß zahlreiche Verleger nicht bloß innerhalb der Partei den Augenblick des Freiwerdens der Marxischen Schriften benützen werden, um mit mannigfachen, kaum immer im Interesse der Partei liegenden und den Ansprüchen der Wissenschaft entsprechenden Ausgaben herauszukommen. Wir erinnern nur an die Herausgabe von Langes Arbeiterfrage durch Dr. Grabowsky, die gebührend in der „Neuen Zeit” und in der „Bildungsarbeit” besprochen wurde. ${ }^{78}$

Es liegt ein hohes Parteiinteresse, auch politische und wissenschaftliche Verpflichtungen vor, daß die Werke von Karl Marx nach Ablauf der Schutzfrist in würdigen Ausgaben, die allen wissenschaftlichen Ansprüchen entsprechen, herausgegeben werden, und daß auch Ausgaben gesichert werden, die der breiten Masse der Arbeiter das Verständnis von Karl Marx näher bringen. Wir zweifeln durchaus nicht, daß vom Parteivorstand, wie von den mit ihm im engsten Zusammenhange stehenden Verlagsbuchhandlungen, ${ }^{79}$ das Freiwerden der Marxischen Schriften für künftige Veröffentlichungen ins Auge gefaßt wurde. Wir glauben aber, $\mathrm{da}$ es kaum eine Parteifrage gibt, in der eine öffentliche Diskussion erforderlicher wäre, wie über das, was im Interesse der Partei und ihres Ansehens und in Wahrung des Andenkens von Karl Marx und zur Durchdringung des Proletariats mit den Lehren von Karl Marx zu geschehen habe. Es handelt sich hier um eines der höchsten Güter der Partei, um Interessen, die im besten Sinne von allen vertreten werden müssen, die

a 4 S. Mschr. (Durchschlag) mit hschr. Korrekturen von A. Braun, Dossier Parteiarchiv (Div. III/I), IISG

${ }^{78}$ F. A. Lange, Die Arbeiterfrage. Ihre Bedeutung für Gegenwart und Zukunft, neu bearb. und hrsg. von A. Grabowsky, Leipzig o.J. [1910]; diese Ausgabe des Verlages Kröner wurde als unkontrollierbare Kompilation aus verschiedenen Auflagen mit stillschweigenden Kürzungen und Änderungen des Herausgebers vernichtend rezensiert in der Neuen Zeit, Jg. 28 (1909-10), Bd 2, S. 559f., und in Bildungsarbeit. Blätter für das Bildungswesen der deutschen Sozialdemokratie in Ósterreich, Jg. 2 (1910-11), S. 20. Bei Vorwärts erschien im gleichen Jahr nach der 2. Auflage von $1870 \mathrm{~F}$. A. Lange, Die Arbeiterfrage, mit Einl. und Anm. hrsg. von F. Mehring (Sozialistische Neudrucke, 4), Berlin 1910.

${ }^{79}$ Die Verlage J. H. W. Dietz Nachf. in Stuttgart und Buchhandlung Vorwärts in Berlin. 
von der Bedeutung von Marxens Werken für den Befreiungskampf des Proletariats überzeugt sind.

Als Parteischriftsteller, die stets bestrebt waren, im Geiste von Marx auf die Arbeiterklasse zu wirken, glauben wir, daß es schon heute notwendig ist, sich mit dem zu beschäftigen, was aus Anlaß des Freiwerdens der Marxischen Werke zu geschehen habe. Die Zeit drängt, weil gute Ausgaben längerer Vorbereitungen bedürfen, wir glauben auch, daß es zweckmäßig wäre, bekanntzumachen, was der Parteivorstand in der Sache zu tun gedenkt, besonders deshalb, weil sonst die einzelnen Parteibuchhandlungen Ausgaben unternehmen und vorbereiten würden, die eine kaum wünschenswerte Konkurrenz gegen eine einheitliche, planmäßig geleitete Herausgebertätigkeit der Marxischen Werke bilden würden. Wir glauben schließlich, daß die Bekanntgabe der Absichten des Parteivorstandes über die notwendigen Marxausgaben den Anlaß zu einer Diskussion geben könnte, die wertvolle Anregungen zeitigen würde.

Wir denken uns ein Komite, vom Parteivorstande eingesetzt, das entsprechend den endgültigen Beschlüssen des Parteivorstandes, eventuell des Parteitages, die Herausgabe der Marxischen Schriften leiten soll. Wir dachten, daß dieses Komite, dem das Recht der Kooptation eingeräumt werden könnte, aus den Genossen Bebel, Cunow, Dietz, Hilferding, Kautsky, Mehring und Rjasanoff bestehen könnte. Wir glauben, daß dieses Komite den Plan der Herausgabe der Werke von Karl Marx im einzelnen feststellen ${ }^{b}$, bei der Durchführung überwachenc, die Aufgaben der Herausgabe unter sich verteilen, beziehentlich Außenstehende mit diesen Arbeiten betrauen soll.

Die Aufgaben, die der Partei nach dem Freiwerden der Marxischen Schriften erstehen, sind vor allem wissenschaftlicher Natur. Es erscheint uns notwendig:

I. eine allen wissenschaftlichen Anforderungen entsprechende, absolut vollkommene, systematisch geordnete, mit den Manuskripten und den verschiedenen Ausgaben der Marxischen Schriften verglichene, mit Einleitung und umfangreichen Registern versehene Gesamtausgabe der Werke von Marx zu veranstalten.

Il. eine allen wissenschaftlichen Anforderungen entsprechende Biographie von Marx, eventuell eingegliedert in die Gesamtausgabe der Werke, zu veröffentlichen.

III. zu erwägen, ob nicht die Werke von Marx und Engels gemeinsam

b Im Original festzustellen

c Im Original zu überwachen 
herausgegeben werden sollten, wegen des engen persönlichen, parteigeschichtlichen, wissenschaftlichen Zusammenhanges dieser Werke, bei denen zum Teil Zweifel obwalten, ob Marx und Engels oder beide als Verfasser zu betrachten sind.

IV. neben den wissenschaftlichen Verpflichtungen ergeben sich die nicht minder wichtigen, Marx in den für die Massen bestimmten Ausgaben seiner Werke dem Verständnisse der Arbeiter näherzubringen. Diese Ausgaben sollen in würdigem Gewande als systematische Sammelbände der sachlich zueinander gehörenden Werke erscheinen:

1. in erster Linie wird man eine Volksausgabe des ersten Bandes des „Kapitals” ersehnen, eine Ausgabe, in der alle fremdsprachigen Bezeichnungen und Zitate übersetzt sind, und in der Anmerkungen die Feststellung von Marx, so zum Beispiel über den Arbeiterschutz, weiterführen. Die Ausgabe soll mit einem Register, mit einer biographischen Einleitung und mit einer Anleitung zum Studium des ersten Bandes des „Kapitals” versehen sein.

2. dann erscheint uns als Einführung der Arbeiter in die Gedankenwelt von Marx besonders wichtig die Sammlungen [!] aller Ausführungen von Marx, insbesondere im „Kapital”, über die Wirtschaftsgeschichte. Die große Bedeutung von Marx als Wirtschaftshistoriker würde so den Arbeitern zur klaren Erkenntnis gebracht werden, sie würden ein leicht lesbares, ein in hohem Maße anziehendes und zum weiteren Studium von Marx anregendes Werk erhalten, das ausschließlich aus Ausführungen von Marx bestehen würde, sich lediglich in der Sammlung und Gliederung des Stoffes als Tätigkeit des Herausgebers ergeben würde.

3. dann denken wir an die Sammlung kleiner Schriften von Marx, in die aber auch einzelne Abschnitte oder Absätze des „Kapitals” einverleibt werden sollten. So Bände:

a) über die materialistische Geschichtsauffassung,

b) über die Klassenkämpfe und über die Revolutionen im 19. Jahrhundert,

c) über die wirtschaftliche Theorie,

d) politische Abhandlungen,

e) seine Ausführungen über die wirtschaftlichen Zustände und sozialpolitische Leistungen und Aufgaben.

Wir sind überzeugt, daß im Kreise des Komites, das wir gerne eingesetzt sehen möchten, wie im Parteivorstande, wie auch in der Gesamtpartei, unsere Vorschläge gewürdigt, verbessert und ergänzt werden mögen. Was wir wollten, war, eine Anregung zu geben. Wir glauben, daß die Veröffentlichung eines Arbeitsplanes der vom Parteivorstande für notwendig erachteten Arbeiten eine Konzentration im wissenschaftlichen wie im Verlegerinteresse, vor allem im Parteiinteresse herbeiführen würde, wäh- 
rend viele an sich gut gemeinte, aber voraussichtlich mangelhafte Arbeiten verhindert würden.

Wien, 1. Jänner 1911

Max Adler, Otto Bauer,

Adolf Braun, Rudolf Hilferding, Karl Renner, N. Rjasanoff

\section{GUSTAV ECKSTEIN AN KARL KAUTSKY}

Lieber Freund!

Wien, 6/1 [19]11.a

Heute bin ich zum erstenmal einige Stunden außer Bett. Hoffentlich kann ich nun bald wieder nach Berlin zurück.

Gestern war Rjasanoff und heute Braun bei mir und erzählten von der „Marxisten-Konferenz”. Ich bin schon sehr neugierig, was Sie zu der ganzen Geschichte sagen. So wie der Plan jetzt vorliegt, würde er wol nicht nur eine Menge Geld erfordern, von der mir doch fraglich ist, ob der Vorstand sich wird auf Jahre hinaus binden wollen und können, sondern er würde auch erfordern, daß einige der besten theoretischen Kräfte sich auf Jahre einer Aufgabe widmen, die zum großen Teil doch nur dekorative Zwecke verfolgt. Eine wissenschaftlich angelegte Gesamtausgabe von Marx' Werken wäre gewiß sehr schön und begrüßenswert; aber ob sie für die Partei eine so unbedingte Notwendigkeit wäre, erscheint mir fraglich. Es würde wol genügen, wenn man die kleineren, heute noch vielfach verstreuten Sachen in ein paar Bänden vereinigte.

Anders steht es mit der Volksausgabe, die wirklich ein Gebot der Partei ist. $^{80}$ Leider konnte ich an jener Konferenz nicht teilnehmen, sonst hätte ich das dort vorgebracht, was allerdings wol auch keine Wirkung gehabt hätte.

Etwa Mitte der nächsten Woche hoffe ich doch wieder in Berlin zu sein. Wien wird mich dann wol nicht so bald mehr wiedersehen!

Dem ganzen Hause herzlichsten Gruß!

Ihr ergebener

GE

\footnotetext{
a 4 S. Hschr., Nachlaß Kautsky D X 57

Adreßvermerk beim Datum Linieng. 4.

$80 \mathrm{Vgl}$. Nr 3.
} 


\section{HEINRICH DIETZ AN KARL KAUTSKY}

Lieber Freund Kautsky!

Stuttgart, den 12. Januar 1911.a

Wenn Geist Goldendach ${ }^{81}$ schon soweit ist, dann dürfte eine offiziöse Mitteilung des Tatbestandes nach Wien sogar notwendig werden.

Unsere Parteileitung hat im Prinzip beschlossen, Marx' Kapital bis Ende 1913 herauszugeben. Mit den Vorbereitungen wurde Dietz betraut, der, gleichfalls im Einverständnis mit der Parteileitung, Kautsky als Herausgeber bestellte. ${ }^{82}$

Wie auch Sie hieraus ersehen, bin ich gebunden und kann mich mit etwaigen Wiener Projekten nicht einlassen.

S. 1: Dietz bittet um Stellungnahme zu einer Neuauflage von K. Kautsky, Der Parlamentarismus, die Volksgesetzgebung und die Sozialdemokratie, Stuttgart 1893, die 1911 als $\mathrm{Nr} 12$ der Kleinen Bibliothek unter dem Titel Parlamentarismus und Demokratie erfolgte.

\section{FRANZ MEHRING AN KARL KAUTSKY}

Steglitz, 16. Januar $1911^{\mathrm{b}}$

S. 1-4: Mehring begründet die Ablehnung eines Beitrages von Eckstein für das Feuilleton der Neuen Zeit aus Raumgründen. Eckstein wollte anscheinend erwidern auf F. Mehring, ,Neu-Lamarckismus und mechanischer Materialismus", in: Neue Zeit, Jg. 28(1909-10), Bd 2, S. 593-602.

In der anderen Sache bin ich vollkommen mit Ihnen einverstanden und habe mich schon vor Ihnen in ganz gleichem Sinne zu Hilferding ausgelassen. Ich habe mich nur bereit erklärt, gelegentlich über die Sache zu reden, was ja nichts schaden kann, wenn auch nichts dabei herauskommen wird. Mit den besten Grüßen von Haus zu Haus

Ihr

F. Mehring

a I S. Hschr. auf Kopfbogen des Verlages, Nachlaß Kautsky D VIII 460 Auszug: S. I

b 4 S. Hschr., Nachlaß Kautsky G 7, 116

Auszug: S. 4

${ }^{81}$ Dietz fürchtete, Rjazanov betrachte bisher erhaltene Vorschüsse als durch seine Forschungsarbeiten erledigt, meinte aber, ,könnte ich ohne Weiteres über Parteimittel verfügen, würde ich kein Wort sagen und es darauf ankommen lassen, ob u. wann G. die ausgegrabenen Schätze auf den Tisch des Verlegers niederlegt. G.s. Schaden wäre es nicht, denn dann könnte ich ihn materiell einreihen. Jetzt steht er unserer Parteikasse gegenüber als Geist.” An K. Kautsky, 8.1.1911, Nachlaß Kautsky D VIII 459.

${ }_{82}$ K. Marx, Das Kapital. [...] Erster Band. [...] Volksausgabe, hrsg. von K. Kautsky, Stuttgart 1914 (weiter Volksausgabe). 
5. ADOLF BRAUN AN KARL KAUTSKY

Wien, 18.I.1911 a

S. 1-12: Braun behandelt das problematische Verhältnis zwischen Marxisten im allgemeinen und der Neuen Zeit im besonderen einerseits und den Gewerkschafien andererseits, das er durch seinen Artikel (wohl A. Braun, „Gewerkschafiliche Verfassungsfragen". in: Neue Zeit, Jg. 29, 1910-11, Bd 1, S. 662-70) verbessern helfen will. Die Affinität deutscher Gewerkschaftsbeamter zum Revisionismus sei weniger strukturell bedingt, als durch Inkompeten: und Ungeschicklichkeit auf marxistischer Seite verursacht. Braun nennt Pannekoek (der kurz vorher das Gegenteil behauptet hatte: ap., ,Gewerkschaftliche Demokratie”, in: [ap.-Korrespondenz.] $\mathrm{Nr}$ 150. 17.12.1910) und die von ihm ausgelöste Polemik, sowie Rosa Luxemburg. Revisionistischerseits werde hier, besonders von den Sozialistischen Monatsheften. geschickt angeknüpft.

Die Geheimnistuerei mit den Marxveröffentlichungen halte ich für durchaus verfehlt. Man kann es nicht als ein Geheimnis sichern, daß Marxens Werke im März 1913 den Urheberschutz verlieren. Das ist eine Sache, mit der sich zahlreiche Buchhändler beschäftigen, ja die Anregung, die von uns aus Wien ausging, hat ihre letzte Wurzel in der Anfrage eines Buchhändlers bei mir, was er denn aus Anlaß des Freiwerdens der Marxischen Schriften tun solle. Gerade wenn die Partei all das, was sie aus Anlaß des Aufhörens des Urheberschutzes zu tun beabsichtigt, ankündigen würde, würde sie einer wilden, ungeregelten und wertlosen Massenproduktion den Hemmschuh anlegen. Das habe ich mit aller Entschiedenheit in Wien vertreten, deswegen wollte ich in Form eines offenen Briefes an Dich einen Zwang ausüben, um diese ,,beste Politik der Geheimnistuerei” unmöglich zu machen. Aber diese energische Form wurde von meinen Kollegen nicht beliebt, und so können wir sicherlich damit rechnen, daß in Frankfurt und in Dresden, in Leipzig und in Nürnberg, ja sogar vielleicht in München Leute das Geschäft werden machen wollen, für stillstehende Maschinen ist Marx immer noch ein gutes Geschäft. Ich wäre sehr entschieden dafür, wenn man sich in Deutschland zu nichts anderem als zu der an sich ja sehr erfreulichen Herausgabe des 1. Bandes von Marx entschließt, im Kampf diese Diskussion vom Zaune zu brechen. Ich habe zwar in Wien den Standpunkt mit aller Entschiedenheit vertreten. daß die Redaktion des Kampf der Neuen Zeit in dieser Frage unbedingt den Vortritt zu lassen hat, wenn aber die Neue Zeit zu dieser so erheblichen

a I4 S. Mschr. mit hschr. Korrekturen und I S. Hschr. auf Kopfbogen Der Kampf, NachlaB Kautsky D VI 312

Auszug: S. $12 \cdot 14$ 
Frage des Marxismus schweigt, so glaube [ich], daß uns die Verpflichtung erwächst, das Wort zu nehmen, worauf dann die Neue Zeit natürlich zur Stellungsnahme veranlaßt werden würde. Ich bitte das doch noch einmal zu erwägen und vielleicht, wenn Du willst mit Benutzung dieses Briefes, hierüber mit Bebel ${ }^{83}$ und dem Parteivorstande und den andern in Betracht kommenden Genossen zu sprechen.

Dich und die Deinen grüßt aufs Herzlichste

Dein

Adolf

S. 15: Postskript über eine Polemik der Holzarbeiter-Zeitung gegen Pannekoek und Brauns Korrespondenz mit deren Redakteur, dessen Antwort er beilegt.

\section{ADOLF BRAUN AN KARL KAUTSKY}

Wien, 18.I.1911.a

\section{Lieber Karl!}

Eine halbe Stunde, nachdem ich den umfangreichen Brief in den Briefkasten versenkt hatte, erhielt ich von Rjasanoff Einblick in Dein Schreiben. Ich möchte mir nur einige private Bemerkungen zu Deinen Ausführungen erlauben. Was Du über die Ausgabe des 1. Bandes des Kapitals schreibst, ist sehr interessant. Sicherlich wäre mit einem guten Register, durch das auf viele Zusammenhänge aufmerksam gemacht würde, viel genützt, zum Teil die Aufgaben eines Kommentars erfüllt. ${ }^{84}$ Die Vorrede über die Art, wie man das Kapital am besten studiert, welche Schriften man vor ihm lesen soll, würde sicher, falls Du diese Arbeit nicht übernehmen willst, Otto Bauer in bester Weise ausarbeiten.

Ich glaube, daß Du Dir über die Gesamtausgabe von Marx zu große Schwierigkeiten vormalst. Vor allem glaube ich, daß diese Edition im Plane und im Umfange festgestellt werden müßte. Kennt man annähernd

a 4 S. Mschr. mit hschr. Korrekturen auf Kopfbogen Der Kampf, Nachlaß Kautsky D VI 313

83 Bebel kehrte vor dem 21.1.1911 nach Berlin zurück, vgl. A. Bebel an L. Kautsky, 21.1.1911, Bebels Briefwechsel mit Kautsky, a.a.O., S. 252.

${ }^{84} \mathrm{Zu}$ den Vorstellungen über die Gestaltung der Volksausgabe vgl. $\mathrm{Nr} 7$. Ein Kommentar war anscheinend von verschiedenen gewünscht und von Kautsky abgelehnt worden, was Dietz kommentierte: „Sie haben recht. mit Marx' Kapital geht's wie mit ,grundlegenden' religiösen Schriften. Über die ,dunklen' Stellen wird eine UUbereinstimmung selbst unter Freunden nicht zu erzielen sein. Verhehlen will ich aber nicht, daß unsere ,Kapital'-Ausgabe gerade durch einen Kommentar einen hohen Reiz erhalten würde. Der Kommentar kann aber auch leicht den Herausgeber auf Wege führen, auf denen er weiter gehen müßte, als ihm lieb sein kann. So wird wahrscheinlich auch hier in der Beschränkung sich der Meister zeigen.” An Kautsky, 8.1.1911, a.a.O. 
Bogen- und Bändezahl, so wird man schon mit Rücksicht auf die überaus geringe Zahl von Herausgebern, die, was Fähigkeiten und verfügbare Zeit anlangt, in Betracht kommen, mit einer nicht geringen Anzahl von Jahren für die Fertigstellung rechnen müssen. Dadurch werden sich die Ausgaben für diese Herausgabe auch auf eine erhebliche Zeit verteilen, so daß für die deutsche Partei finanzielle Erwägungen - meiner unmaßgeblichen Meinung nach - nicht in Betracht kommen dürften. Im übrigen scheint mir doch das Testament von Engels die Partei auch bedacht zu haben, ${ }^{85}$ so daß auch hieraus Mittel für diesen Zweck geschafft werden könnten. Würde das Komitee, das wir eingesetzt wünschen, den Plan der Gesamtausgabe der Marxischen Werke aufstellen, wobei man ja mit einem oder zwei Bänden ,Varia” für künftige Entdeckungen vorsorgen könnte, so könnten in ungeregelter Folge nach und nach diese Werke erscheinen. Ich bin durchaus nicht der Meinung, daß man auf den Privatdozenten einer künftigen sozialistischen Universität warten müsse, um diese Arbeit auszuführen. Ich berücksichtige alle Schwierigkeiten, die eine Herausgabe der Werke von Karl Marx mit sich bringt, aber ich meine, daß es sich hier um eine so wichtige Verpflichtung der Partei handelt, daß man sich ihr nicht entziehen soll und darf. Dabei verstehe ich ganz wohl, daß Du Dir nicht diese große Last auch noch aufbürden willst. Was man von Dir wünscht, ist lediglich eine oberste Leitung des Komitees und eine Oberaufsicht und Gutachtertätigkeit bei der Herausgabe. Das Ausscheiden von Personen, die unseres Erachtens nicht in Betracht für die Herausgabe kommen, soll natürlich auch aus Deiner leitenden Stellung folgern.

Sämtliche Werke von Marx der Forschung und der Partei zugängig zu machen, ist, glaube ich, auch eine Gegenwartsaufgabe. Was der Partei vielfach nottut, ist die neuerliche Befruchtung durch die Marxische Gedankenwelt, vor allem vielfache Formen der Anregung an die Alten und an die Jungen sich mit Marx neuerdings oder zum erstenmale zu beschäftigen. Ich glaube man sollte nicht warten, bis irgend ein unternehmungslustiger Verleger die Aufgaben übernimmt, die uns zu erfüllen bestimmt sind. Wenn man heute in Verlegerkreisen sich ein Geschäft davon verspricht, Thünens Isolierten Staat in einer verhältnismäßig sehr billigen Ausgabe zu veröffentlichen, ${ }^{86}$ so wird sich auch einer finden, der vor der

${ }^{85}$ Eine zumindest globale Kenntnis der finanziellen Verfügungen von Engels kann in Wien vorausgesetzt werden, da die Arbeiterzeitung in den 1890-er Jahren wiederholt aus diesen Mitteln unterstützt worden war; vgl. z.B. $\leadsto$ A. Bebel an V. Adler, 18.9.1895, in: Adler-Briefwechsel, S. 187. Einen Auszug aus dem Testament erwähnt Braun an Kautsky, 20.3.1909, a.a.O.

${ }^{86}$ Im Verlag von Gustav Fischer erschien J. H. von Thünen, Der isolierte Staat in Beziehung auf Landwirtschaft und Nationalökonomie, [ . . . ] eingel. von $\mathrm{H}$. Waentig, Jena 1910, zum Preise von 7,- bzw. geb. 8,- Mk. 
Herausgabe von Marx' gesammelten Werken nicht zurückschreckt. Dann aber wird es uns wirklich finanziell unmöglich sein, daneben noch mit einer zweiten Ausgabe zu kommen. Ich bin also der Meinung, daß hier eine Pflicht für die Partei vorliegt, daß ihre Erfüllung der Partei etwas finanziell unmöglich[es] nicht zumutet, daß die Verteilung der Ausgaben auf eine Reihe von Jahren die Herausgabe ermöglicht und daß auch die wissenschaftlichen Kräfte für diese Aufgaben freizumachen sind.

Aber ich meine, daß daneben unsere andern Vorschläge nicht unbeachtet bleiben sollen, so vor allem die Herausgabe der einzelnen Sammelbände von kleinen Schriften und dazugehörigen Ausführungen in den größeren Werken, geordnet nach Hauptgruppen der wissenschaftlichen $u$. politischen Arbeit von Marx. Diese Sammelbände könnten wohl ohne große Schwierigkeiten für den März 1913 fertiggestellt werden.

Von größter Wichtigkeit erscheint mir, daß das was geschehen soll auch bekannt gemacht wird. Es erscheint mir dies eine Pflicht gegenüber allen denen, die sich geschäftlich oder als Herausgeber mit dem Gedanken der Veröffentlichung von Marxischen Schriften über die Zeit ihres Freiwerdens tragen. Ich glaube, daß die Partei den Anspruch hat zu wissen, was geplant wird, ich glaube, daß das auch geschäftlich klug wäre und daß eine derartige vorläufige Veröffentlichung die beste Reklame wäre, um einen starken Absatz der Schriften in den geplanten Ausgaben zu sichern.

Hilferding schrieb mir, daß Ihr am (letzten) Sonntag ${ }^{87}$ eine Beratung haben werdet über die von uns gemachten Vorschläge. Bisher hatte er uns aber über diese Zusammenkunft nicht berichtet. Ich hoffe, daß auch Bebel von unsern Vorschlägen etwas zu hören bekommt und daß der Parteivorstand sich auch offiziell mit der Angelegenheit befassen wird.

Wenn Du schreibst, daß sich in der Beschränkung der Meister zeigt, so wende ich dagegen ein, daß das, was wir vorgeschlagen haben, meinem Erachten nach in keiner Weise im Widerspruche steht, in dem was finanziell und wissenschaftlich geleistet werden könnte, ich glaube auch nicht, daß irgendwie eine Arbeit unterlassen werden müßte, weil diese Ehrenaufgabe in Angriff genommen wurde.

In der Hoffnung, daß Du meine Ausführungen freundlichst entgegennimmst, bleibe ich

Dein

Adolf 
7. DAVID RJAZANOV AN KARL KAUTSKY

20 Jänner $1911^{\mathrm{a}}$

Lieber Freund,

Zuerst einige Worte in Frage des „Kapitals”. Aus Ihrem Brief geht hervor - vielleicht habe ich nicht gut verstanden - daß es sich nur um den Ersten Band handelt. Für eine Volksausgabe ist nur dieser geeignet. Eine gleichzeitige Ausgabe des ersten zusammen mit den 2 und 3ten kann separat erscheinen oder im Rahmen der Gesammelten Werke.*

Ein Kommentar ist überflüssig. Er kann als eigenes Werk eines Marxisten erscheinen in der Art des Diehl-schen zum Ricardo. ${ }^{89}$ Aber für diese Volksausgabe wäre notwendig einige Ergänzungsnoten zu machen Literaturangaben, wo Marx Bücher zitiert, die den ,gegenwärtigen” Stand der Technischen Entwicklung zeigen; Neue Daten aus der Geschichte der Fabrikgesetzgebung, der Technik, der Großindustrie; Ich bin bereit diese Arbeit auf mich zu nehmen, sowie die Einleitung, wie man das Kapital am besten studirt. Ich will dabei verwerten meine jetzt mehr als zwanzigjährige Erfahrungen als Lektor über „Kapital” und Popularisator für die Arbeiter. Sehr zweckmäßig wäre es, auch diese Einleitung für die Gewerkschafter interessant zu machen, und für diese jene Partien hervorzuheben, die für die "Praktiker” von eminenter praktischen Wichtigkeit sind. Überhaupt konnte man sehr leicht in Noten und kleinen Ergänzungen eine Menge Thatsachen und Erscheinungen registrieren, um den Arbeitern aus dieser Ausgabe eine Encyclopädie der kapitalistischen Produktionsweise [zu] machen. Als Nachwort konnte man ein Literaturnachweis für weitere Studien geben und einen kurzen Abriß des Inhalts des zweiten und dritten Bände oder eine Einleitung zum Studium, den Engels'schen Brief an V.

* Daß es auch vom kaufmännischen Standpunkt eine profitbringende Sache ist, beweist die unlängst erschienene 6 Auflage des Ersten und neue Auflagen des 2, und 3 Bände. ${ }^{88}$

a 4 S. Hschr, Nachlaß Kautsky D XIX 327

Auszug: S. 1 -4

Adreßvermerk beim Datum Anton Menger Bibliothek / N. Riasanov / Wien I Seiler Stätte 7 I

88 K. Marx. Das Kapital, Bd 1 erschien bei Meißner 1909 in 6., Bd 2 und 3 in 4. bzw. 3. Auflage im Jahre 1910.

89 K. Diehl, Sozialwissenschaftliche Erläuterungen zu David Ricardo's Grundgesetzen der Volkswirtschaft und Besteuerung, 2 Bde, Leipzig 1905, erschienen als Bd 2 und 3 zu D. Ricardo, Grundgesetze der Volkswirtschaft und der Besteuerung, übers. von $E$. Baumstark. 2. Aufl., Leipzig 1877; Diehls Bände ersetzten Baumstarks Kommentarband von 1838. Als Vorbild für eine Volksausgabe scheint dieses bibliographische Monstrum weniger glücklich. 
Adler als Grundlage nehmend. ${ }^{90}$

Ein Sachregister muß das Theoretische und Praktische gleichmäßig behandeln, sodaß nicht nur der „Politiker”, sondern auch der „Gewerkschaftler", leicht und bequem alles zur Hand hat, was er für seine Zwecke braucht. Den Namensregister kann man mit Biographischen Notizen „,verschönern". 91

Was die fremdsprachlichen Noten betrifft, so kann man jetzt eine Menge nach betreffenden deutschen Ausgaben zitieren, und wo das nicht der Fall ist, kann diese Arbeit von Eckstein am besten gemacht werden ${ }^{92}$ Ein Paar italienische Zitaten können auch leicht übersetzt werden.

Obwohl Sie die Arbeit des Vergleiches des Text mit der französischen Ausgabe auf sich nehmen, ${ }^{93}$ werde ich auch Ihnen bei dieser Arbeit behilflich sein. Ich habe sie einmal für Selbstgebrauch durchgemacht. Ich bin nur dagegen, daß man den Text von Fremdworten reinigt. Es ist schon besser am Schluß ein kleines Wörterbuch zu machen oder in Noten verschiedene Anglizismen zu berichtigen. ${ }^{94}$

Eines ist unbedingt notwendig: Sie müssen eine Vorrede schreiben, wo Sie die Bedeutung des „Kapitals” für die Wissenschaft und die Arbeiterbewegung auseinandersetzen. ${ }^{95}$

Im ganzen muß man damit rechnen, daß die Volksausgabe um 4-5 Bogen stärker wird, vielleicht noch mehr. ${ }^{96}$ Dann kann man den Ersten Band wie den dritten in zwei Hälften teilen.

90 F. Engels an V. Adler, 16.3.1895, MEW, Bd 39. S. 436-38.

91 Der von Rjazanov bearbeitete Registerteil der Volksausgabe umfaßt 68 Seiten, enthält eine eigene sechsseitige Einleitung, ein Verzeichnis der zitierten Werke, ein Namensregister mit kurzen biographischen Mitteilungen und ein Sachregister; die Bedeutung des Werkes für Theorie und Praxis der Gewerkschaften betont Rjazanov in seiner Einleitung. S. 706.

92 Für die Textgestaltung der Volksausgabe vgl. Kautskys Vorwort des Herausgebers. hier zur Übersetzung und Überprüfung fremdsprachlicher Zitate durch Eckstein S. xxxf.

93 Zur Berücksichtigung des Textes der französischen Kapital-Ausgabe von 1872-75 in der Volksausgabe ebd., S. XV-Xvilı.

91 Kautsky entschied sich hier anders, sein „Kampf gegen die Fremdworte hatte vor allem in einer Ausmerzung von Anglizismen zu bestehen" und erfolgte. allerdings vorsichtig, im Text. Zur Begründung vgl. ebd., S. xxI-XxvI.

95 Diese Auseinandersetzung fehlt in Kautskys Vorwort, Rjazanovs Vorstellungen sind in seiner Registereinleitung bei der Begründung der Anlage des Sachregisters erkennbar; vgl. ebd., S. 704-06.

96 Die Volksausgabe zählt XLVIII +768 S. gegenüber der 6. Meißnerschen Auflage von 1910 mit XXXII + 739 S. Die Prognose ist aber nur scheinbar unterschritten. Vorwort des Herausgebers und Register umfassen 91 Seiten und ersetzten nur 9 S. Einleitung von Engels, der größere Blattspiegel und gedrängtere Satz der Volksausgabe hielten deren Wachstum in Grenzen. 
Es ist selbstverständlich, daß ich bis Juli 1912 dieser Arbeit meine ganze Arbeitszeit nicht widmen kann. Den Winter 1911-1912 werde ich in Berlin verbringen und dann können wir den Plan dieser Arbeit vollständig ausarbeiten. Aber vieles kann noch bis Juli 1912 gemacht werden, und von da an werde ich mich darauf konzentrieren sodaß der Band im März 1913 erscheinen kann.

Und jetzt komme ich zum Punkt 2. Ich theile nicht Ihre Bedenken wegen der Gesamtausgabe. Aber - und das habe ich den „Kämpfern” gesagt der Zeitpunk[t] ist nicht günstig gewählt um auf den Parteivorstand einzuwirken. Vielleicht nach dem Wahlsieg wenn die Partei 120 Vertreter im Reichstag bekommt und 4000000 Stimmen $^{97}$ und wenn nicht der gesamte Parteivorstand $b$ von Janytscharen gehängt wird, konnte man noch den Pfannkuch, ${ }^{98}$ Fischer ${ }^{99}$ und andere dazu bringen, daß sie diese Ausgabe wirklich als eine Ehrensache der Parteib betrachten. Und nur nach den Wahlen kann man nötigenfalls den Acheront ${ }^{100}$ in Bewegung setzen. Früher wird es schwer sein. Und eben deshalb will ich nicht weiter über diese Frage diskutieren.

S. 4: Rjazanov begrüßt die Aufnahme der Artikel von J. Sassenbach und A. v. Elm (.,Deutscher Heimarbeitertag” bzw. „Massen und Führer”, in: Neue Zeit, Jg. 29, 1910-11, Bd 1, S. $489-93$ bzw. 521-26) als Mittel, die Zeitschrift von Pannekoeks Unkenntnis in Gewerkschaftsfragen abzusetzen. Notwendige Polemik gegen die „Führer" müsse verdeckt, als Polemik gegen englische Gewerkschaftspraxis, geführt werden.

b Von Parteivorstand bis Partei durch Randstreichung mit Blaustift hervorgehoben; von konnte bis Partei mit Blaustift unterstrichen; als eine Ehrensache der Partei mit Rotstift unterstrichen

97 Das Ausmaß des sozialdemokratischen Wahlerfolgs von 1912 wird in der Prophezeiung von Rjazanov unterschätzt, was die im ersten Wahlgang tatsächlich erreichten 4.250.399 Stimmen angeht, und überschätzt angesichts von 110 tatsächlich errungenen Mandaten: vgl. Die Reichstagswahlen von 1912, Heft 2 (Statistik des Deutschen Reichs, Bd 250/2). S. 7 und 9.

98 Wilhelm Pfannkuch (1841-1923), damals Mitglied des Parteivorstandes.

99 Richard Fischer (1855-1926), damals Geschäftsführer des Verlages Buchhandlung Vorwärts.

100 Vergil, Aeneis, Buch VII, Vers 312: „Flectere si nequeo superos, Acheronta movebo”; für Lesung und Deutung danke ich nicht G. Büchmann, sondern meinem Kollegen J. Kloosterman. 


\section{FRANZ MEHRING AN KARL KAUTSKY}

Steglitz, 23. Januar 1911a

S. 1: Mehring ist besorgt über eine Erkrankung von Minna Kautskv.

$\mathrm{Da}$ eine Besprechung über die Marx-Ausgabe mit dem von Ihnen angegebenen Zweck sehr nützlich sein wird, glaube ich auch, und ich bin bereit, mich daran zu beteiligen.

Mit besten Grüßen von Haus zu Haus

Ihr

F. Mehring.

\section{FRANZ MEHRING AN KARL KAUTSKY}

Lieber Freund!

Steglitz, 16 Februar 1911b

Das Memorandum der Wiener Genossen, das ich wieder beifüge, war mir noch nicht bekannt. Ich stehe der Sache aber noch ablehnender gegenüber, als früher, seitdem ich durch Dietz erfahren habe, daß Ihnen eine populäre Ausgabe des ersten Bandes des Kapital durch den PV übertragen worden ist. Damit ist das zunächst Notwendige geschehen. Die anderen Pläne der Wiener Genossen sind meines Erachtens für absehbare Zeit unausführbar, und das geplante Komitee würde zu einer wenig beneidenswerten Rolle verurteilt sein, womit ich mich übrigens einer Unterhaltung über die Sache nicht versagen will.

S. 1-2: Redaktionstechnisches, Gruß und Unterschrift.

10. ADOLF BRAUN AN KARL KAUTSKY

Wien, 16.2.1911.c

S. 1-3: Braun betrachtet die Folgen von Pannekoeks Artikel .,Gewerkschaftliche Demokratie" als ,bedauerliches Unheil" und vermutet hinter der prompten

a I S. Hschr., Nachlaß Kautsky G 7,117

A uszug: $S$. 1

b 2 S. Hschr., Nachlaß Kautsky D XVII 337

Auszug: $S .1$

c 4 S. Mschr. mit hschr. Korrekturen, Familienarchiv Kautsky, Port. 1, Mappe 4 Auszug: S. 4 
Reaktion der Gewerkschaftsblätter Steuerung. Er hat gegen die Verallgemeinerung der Polemik gegen die marxistische Richtung bei den Redaktionen von Holzarbeiter-Zeitung, $M$. Kavser, und Grundstein, A. Winnig, mit gewissem Erfolg protestiert und schickt Abschrift der Antwort von Winnig.

Wir würden sehr gerne etwas erfahren über den Gang der Marx-Publikationen. Ich wenigstens bin der Meinung, daß man zum mindesten unsere Vorschläge zur Diskussion stellen soll, wenn man sich zu unseren Anträgen nicht entgegenkommend stellen will. Ich glaube nicht, daß man die Angelegenheit einfach durch Ignorieren oder Nichtbeantworten unserer Denkschrift erledigen kann. Ich beabsichtige, die meines Erachtens nach bedeutungsvolle Frage zur Diskussion zu stellen, ich halte die Neue Zeit für den geeignetsten Ort, solltest $\mathrm{Du}$ aber zu meinem lebhaftesten Bedauern davon absehen müssen, so werden wir die beschränktere Öffentlichkeit des Kampf in Anspruch nehmen. Mit den besten Grüßen auch an die Deinigen bleibe ich

Dein Adolf

\section{DAVID RJAZANOV AN KARL KAUTSKY}

Lieber Freund,

[Paris,] 14 Dez [1911]

Was Sie mir über Lafargues schreiben bestätigt auchb meine Vermutungen. Ich habe nie geglaubt, daß beide Millionen haben, aber nur in diesem Sommer habe ich zufällig erfahren, daß sie nichts haben. Ich war wieder einige Male dort und einmal bin ich zwei Tage geblieben. Wir haben sehr oft über den Nachlaß gesprochen. Laura wollte die Manuskripte dem Britischen Museum vermachen, und ich habe wiederholt versucht ihr zu beweisen, daß diese Weise die Dankbarkeit Ihres Vaters versus Museum zu bezeugen nicht zweckmäßig ist. Und ich glaube, in diesem Zusammenhang habe ich auch erwähnt, daß diese Manuskripte (der größte Theil ist bei Ihnen) sehr wichtig sind für eine Herausgabe der Sämmtlichen Werke Ihres Vaters, die schon projektirt ist. Die Nachricht hat sie stark aufgebracht und die gewöhnlich so liebenswürdige Laura hat dabei sehr starke Ausdrücke gegen die „Frevler” gebraucht. Darauf habe ich ihr bemerkt, $\mathrm{da} ß$ ich auch dabei bin, daß es ist kein Grund zu glauben, daß diese „Jungen" daraus materielle Vorteile ziehen wollen, weil das Recht auf

a 6 S. Hschr., Nachlaß Kautsky D XIX 295

Auszug: S. $1-5$

b Im Original teilweise kyrillisch aux 
Marx'sche Werke im März 1913 erlischt und jeder bürgerliche Verleger kann dann ruhig eine verunzierte Ausgabe machen. Sie hat sich beruhigt, aber sofort an Paul gewendet, dem sie den Inhalt unserer Unterhaltung mitgeteilt hat. Und ich habe wieder bemerkt, daß ihn diese Nachricht ebenso sehr aufgebracht hat. Er wollte mir beweisen, daß das Recht nur nach 50 Jahren erlischt. ${ }^{101}$ Da der weitere Streit zwecklos war, habe ich ihnen versprochen, das nächste Mal das deutsche Urheberrechtsgesetz mitzubringen. Ich erinnere mich noch sehr gut, daß ich in zwei Buchhandlungen den Versuch gemacht, das Gesetz in Reclamscher Ausgabe zu kaufen, aber es ist mir nicht gelungen. Ich habe nur in der Bibl. Nat. den Text wieder nachgesehen und am nächsten Sonntag, vor meiner Abreise nach London, Ihnen das Resultat mitgeteilt. Sie waren schon ruhiger. Lafargue sprach mehr davon, daß diese Sache wird sehr unangenehm für die Neffen sein. Laura hat mir dabei erzählt, daß in den letzten Jahren die Hamburgsche Ausgaben viel mehr gebracht haben, als man gewöhnlich glaubte, daß der alte Meißner sehr korrekt war und daß der gesammte Ertrag zwischen Ihnen und den Neffen geteilt war.* Von Laura weiß ich auch, daß sie und Paul mit einem reichen Franzosen wegen der Herausgabe in franz. Sprache der Hauptwerke Marx's verhandelt haben und da für sie sehr wichtig die Frage war, welche Rechte hatte der frühere Herausgeber des ,Kapitals”, hat sie mich gebeten, alle diesbezügliche Briefe auszusondern. So habe ich es auch gemacht. (Von London habe ich Ihnen ein englisches Blue Book mit den Texten verschiedener Urheberrechtsgesetze Geschickt)

Es war für mich klar, daß diese Einkünfte aus Marx'schen Werken nur deshalb eine solche große Rolle spielen, weil sie nicht so reich sind, wie man es geglaubt hatte. Die Millionen seiner Mutter, von denen noch Zéro-Mouchard ${ }^{102}$ schrieb, waren noch weniger reell als die Millionen, die

\footnotetext{
* Zwei neue Auflagen des lten Bandes, neue Auflagen des zweiten und dritten und dann noch etwas von Dietz
}

101 Dies traf für die Schutzfristen nach französischem Urheberrecht zu, der internationale „Berner Verband zum Schutze des Urheberrech ts” beruhte aber auf dem Prinzip der formalen Reziprozität, ,.Der Umfang und die Mittel des Schutzes richten sich also im allgemeinen ausschließlich nach den Gesetzen des Landes, in dem der Schutz beansprucht wird." Allfeld, „Urheberrecht”, a.a.O., S. 133 und 131.

i0̄2 „Zéro-Mouchard” ließ sich nicht identifizieren. Zu Spekulationen über das Vermögen der Lafargues im Umkreis der sozialistischen Bewegung und deren Niederschlag in Polizeiberichten vgl. allgemein C. Willard, Le mouvement socialiste en France (1883-1905). Les Guesdistes, Paris 1965, S. 133. Zeitgenössisch führt A. Zévaès, Notes et souvenirs d'un militant, Paris 1913, S. 131, den Wohlstand von Paul Lafargue auf eine mütterliche Erbschaft, den Reichtum auf das Erbe von Engels zurück. Eine Anspielung Rjazanovs auf eine frühere Äußerung des Ex-Guesdisten Zévaès ist möglich. 
ihnen die Marx'schen Bücher gebracht haben. Engels' Geld haben sie vollständig in ihre Villa hineingelegt. So hat sie es mir erzählt. Einkünfte aus diesem Gut, außer dem was es für den Tisch an Gemüse und Geflügel gab, hatten sie nicht. Schon die verstorbene Woynarowska**103 erzählte mir etwas, und trotz ihrer lebhaften Imagination, hat sie sehr vieles richtig gesehen. Bei allem äußeren „Prunk” waren Lafargues an Geld sehr arm, und es steht für mich außer jedem Zweifel, daß sie für sich nicht ein solches sorgloses Leben führten, als für Gäste. Und ich bin überzeugt, daß die Frage - was weiter - hat, bei den französischen Parteiverhältnissen von denen er mir auch etwas erzählte, beide sehr stark beschäftigt. Die Vermutung, daß er das Geld in einer Börsenspekulation verloren hat, halte ich für eine Grundlose. Wahrscheinlicher ist, daß das wenige, was sie von seiner Mutter hatten, schon lange verausgabt war. Man kann nur vermuten, daß er, der soviele Jahre mit der Not gekämpft hat, hatte jetzt keine Lust mit siebzig Jahren diesen Kampf um das Brot wieder aufzunehmen und $d a ß$ sie alles aufgeboten hat, um ihn von seiner Absicht abzuhalten. Und doch ist die Tat für Laura unerwartet rasch gekommen und nur so kann man erklären, daß sie kein Wort geschrieben hat.

Was den Nachlaß betrifft, so hat er jetzt keine solche Bedeutung. Die wichtigsten Manuskripte sind bei Ihnen, bei Mehring und mir. Auch die wichtigsten Briefe sind bei mir und Mehring. Es sind geblieben eine Reihe Briefe schon von Mehring für den Nachlaß benutzt und Diversa, die keine entscheidende Bedeutung haben. Alles ist schon klassifizirt. (darunter interessante Briefe von Loria, Hyndmann, überhaupt die Pakete mit Briefen von 1874 bis 1881) Wichtig sind noch Marx's Hefte mit seinen Excerpten, aber auch diese geben nichts neues (zwei sind bei mir). Laura sollte noch ihnen ein Paket für mich schicken, aber sie hat es wahrscheinlich nicht getan. (U. And. eine von Marx zusammengestellte oekon Geschichte der 68 und 69 Jahre und 1873)

Vielleicht hatte auch Paul Briefe von Engels und Laura einige von Ihrer Mutter. Von letzteren habe ich nur ein Paar gesehen. Eine Anzahl Briefe von Paul und Laura an Marx habe ich. Und ich glaube daß alles was ich habe und was ich dem Mehring gegeben später dem Archiv einverleibt werden kann, weil man kann es ad Internationalia rechnen, und diese hat

** Ihre Papiere sind auch bei Lafargue. Ich sollte sie mitnehmen, um der Polnischen Partei zu geben, aber ich habe es vergessen.

103 Cesaryna Wanda Wojnarowksa (1858-1911), seit 1879 in der polnischen sozialistischen Bewegung tätig, emigrierte 1883 nach mehrmaligen Verhaftungen in die Schweiz, später nach Paris, und war seit 1894 Mitglied der Auslandsorganisation der SDKP(iL); vgl. F. Tych, ..Listy Róży Luksemburg do Cezaryny Wojnarowskiej (1896-1905)”, in: Z. Pola Walki. Jg. 14 (1971), Nr I. S. 199-201. 
Laura für die Sammlung gegeben. Es ist doch besser, als die Briefe dem Cubanischen Cousin oder dem Jean Longuet, von dem der Paul immer als von einem Idioten sprach, zu geben.

S. 5-6: Rjazanov schickt Manuskript über Marx und Artikel von Marx, .., Revolution in China and Europe". - Über die gegenwärtige Konstellation der russischen sozialdemokratischen Fraktionen, etwa analog zu Dokument 208 in D. Gever, Kautskys Russisches Dossier, Frankfurt usw. 1981, S. 592-94. 